\title{
Robust estimation of planar surfaces using spatio-temporal RANSAC for applications in autonomous vehicle navigation ${ }^{\text {म }}$
}

\author{
Faisal Mufti ${ }^{\mathrm{a}, *}$, Robert Mahony ${ }^{\mathrm{b}}$, Jochen Heinzmann ${ }^{\mathrm{c}}$ \\ ${ }^{a}$ Electrical and Computer Engineering, Center for Advanced Studies in Engineering, Islamabad, Pakistan \\ ${ }^{\mathrm{b}}$ School of Engineering, College of Engineering and Computer Science, Australian National University, Canberra, ACT 0200, Australia

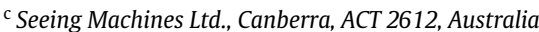

\section{A R T I C L E I N F O}

\section{Article history:}

Received 23 August 2010

Received in revised form

6 August 2011

Accepted 17 August 2011

Available online 12 September 2011

\section{Keywords:}

RANSAC

Time-of-flight cameras

Robustness

Navigation

Detection

Ground plane

Segmentation

Obstacle avoidance

\begin{abstract}
A B S T R A C T
A fundamental problem in autonomous vehicle navigation is the identification of obstacle free space in cluttered and unstructured environments. Features such as walls, people, furniture, doors and stairs, etc are potential hazards. The approach taken in this paper is motivated by the recent development on infra-red time-of-flight cameras that provide video frame rate low resolution depth maps. We propose to exploit the temporal information content provided by the high refresh rate of such cameras to overcome the limitations due to low spatial resolution and high depth uncertainty and aim to provide robust and accurate estimates of planar surfaces in the environment. These surfaces' estimates are then used to provide statistical tests to identify obstacles and dangers in the environment. Classical 3D spatial RANSAC is extended to $4 \mathrm{D}$ spatio-temporal RANSAC by developing spatio-temporal models of planar surfaces that incorporate a linear motion model as well as linear environment features. A 4D-vector product is used for hypotheses generation from data that is randomly sampled across both spatial and temporal variations. The algorithm is fully posed in the spatio-temporal representation and there is no need to correlate points or hypothesis between temporal images. The proposed algorithm is computationally fast and robust for estimation of planar surfaces in general and the ground plane in particular. There are potential applications in mobile robotics, autonomous vehicular navigation, and automotive safety systems. The claims of the paper are supported by experimental results obtained from real video data for a time-of-flight range sensor mounted on an automobile navigating in an undercover parking lot.
\end{abstract}

(c) 2011 Elsevier B.V. All rights reserved.

\section{Introduction}

Navigation [1], path planning [2], and obstacle avoidance [3] are fundamental problems in robotics and autonomous navigation [4]. The importance of the application domain has generated significant effort and techniques, benefitting many research areas, for example, planetary exploration [5,6], indoor [7] and outdoor [8] navigation, ground plane detection [9,10], scene segmentation [11], industrial automation [12], localization of the environment [13], collision free maneuverability in dynamic and urban environments $[14,15]$ to name just a few examples. The task of navigation in industry has classically been approached by either

\footnotetext{
th This work was supported by Seeing Machines Ltd. and the Commonwealth of Australia, through the Cooperative Research Centre for Advanced Automotive Technology.

* Corresponding author.

E-mail addresses: faisal.mufti@ieee.org (F. Mufti), robert.mahony@anu.edu.au (R. Mahony), jochen.heinzmann@seeingmachines.com (J. Heinzmann).

URL: http://www.seeingmachines.com (J. Heinzmann).
}

(a) directly detecting and avoiding obstacles [16,17] or (b) estimating the dominant planar feature (ground plane) and segmenting hazardous features from the detected ground plane $[9,13,18]$.

Vision based techniques to compute safe space in which motion is possible for autonomous navigation has mostly been explored with pure intensity images, using shape [19], motion [20], model fitting [21], stereo vision [22], color cues [8], and stochastic modeling techniques [23], to develop autonomous navigation and obstacle detection algorithms. However, two dimensional spatial visual sensors are limited in high dynamic light environments as well as adverse weather conditions of dust and fog. This can be overcome by using range sensing devices that are robust against texture and limited visibility. Range devices can operate in complex environments such as at night [24] and in underground mines [25] and are ideal for navigation applications in autonomous mobile robotics and automotive [26-29].

A number of methods for ground and planar surface estimation using three dimensional range data have been proposed [30-33]. Early work [34] on planar surface segmentation in range images used a split-and-merge algorithm for surface approximation. Jiang and Bunke [35] proposed scan line grouping for planar surface segmentation by treating a complete line segment as 
an atomic entity. Meier and Ade [36], suggested simple linear least squares for plane fitting on 3D data obtained from a range sensor and used this in obstacle detection and tracking algorithms for vehicles. Wang et al. [37] solved plane fitting of noisy range data as a minimization problem obtaining the optimal solution via partial weighted eigen method. Hähnel et al. [38] suggested a region growing method for planar surface approximation. In [39] an approach based on Hough transform representation of a plane for 3D range data obtained from laser scanner mounted on a Segway ${ }^{\circledR}$ Robotic Mobility Platform (RMP) for an outdoor environment is presented. A statistical framework [40] of Expectation Maximization (EM) has also been developed for estimation of planar surfaces (walls, doors, ceilings) [41,42]. The EM approach has been further explored by Triebel et al. [43] using Hierarchical EM and by Lakaemper and Latecki in [44] using extended EM. Other techniques based on computationally expensive semantic or scene constraint approaches $[45,46]$ have been analyzed for segmentation using $1 \mathrm{D} / 2 \mathrm{D}$ laser range or LIDAR (Light-Imaging Detection and Ranging) data [47,18]. These methods work well for dense 3D point clouds but in most cases require registration of scanned laser range data for 3D estimation. Dense range estimation algorithms for highly detailed reconstructions, often suffers from hallucination [48-50] due to the amount of noise and outliers generated by dense stereo matching. However, range data from 3D TOF cameras does not require depth estimation from images [51]. Recent works on plane fitting uses time-of-flight range data [52,53]. Poppinga et al. [52], proposed a plane fitting method on range data using region growing technique from the nearest neighbors followed by sequentially polygonalization to turn regions into surface models.

A well regarded technique for segmentation and robust model fitting of range data is the statistical framework of RANdom SAmple Concensus (RANSAC) [54]. Recently, Nüchter et al. [46] used a combination of Iterative Closest Points (ICP) and RANSAC to extract planar features using range data. Apart from some prior work of the authors [55], existing work that applies RANSAC to 3D data for plane fitting uses single frame data [56-58], or tracks individual data points between frames [59]. In [60], RANSAC plane fitting with Kalman filtering on sequentially obtained range data from LIDAR system is presented. This requires estimation of appropriate magnitude of the parameters of the state transition that may reduce system accuracy. Unlike the laser scanners that require multiple scans, range image devices based on the principle of time-of-flight (TOF) [61] acquire a full 3D image in a single exposure and can be run at video frame rate. In addition, TOF cameras are robust against shadow, brightness and poor visibility, making them ideal for autonomous navigation applications. In recent years various companies have developed time-of-flight sensors, and as the application and demand is growing for such sensors, they are becoming less expensive and available for commercial applications [62].

In this paper, we have formulated a spatio-temporal RANSAC algorithm for planar surface estimation using 3D video data. The TOF camera/sensor provides 3D spatial data at video frame rate and is recorded as a video stream. We model a planar 3D feature comprising two spatial directions and one temporal direction in 4D. We consider a linear motion model for the camera. In order for the resulting feature to be planar in the full spatio-temporal representation, we require that the camera rotation lies in the normal to the ground plane, an assumption that is naturally satisfied for ground vehicles. A minimal set of data consisting of four points is chosen randomly amongst the spatio-temporal data points. From these points, three independent vector directions, lying in the spatio-temporal planar feature are computed. A model for the 3D planar feature is obtained by computing the 4D cross product of the vector directions. The resulting model is scored in the standard manner of RANSAC algorithm [63] and the best model is used to identify inlier and outlier points. The final planar model is obtained as a maximum likelihood (ML) estimation derived from inlier data where the noise is assumed to be Gaussian. The technique has potential applications for ground plane estimation, planar surface segmentation and obstacle detection. By utilizing data from a sequence of temporally separated image frames, the algorithm robustly identifies the planar feature such as the ground plane even when the ground plane is mostly obscured by passing pedestrians or cars and in the presence of walls (hazardous planar surfaces) and other obstructions. In addition, due to the resolution of sparse depth images, we do not deal with hallucination and only the few dominant planes are detected. The fast segmentation of the obstacles is done both in spatial and velocity domains by employing a statistical threshold. The proposed algorithm is simple, does not require correlating data points or hypothesis between image/frames, and is based on $4 \mathrm{D}$ vector product from spatio-temporal set of range data. It is computationally inexpensive without the need of image/feature selection, calibration or scene constraint and is easy to implement in the fewest possible steps as it only requires estimation of normal vector from randomly sampled 4D data.

The paper is organized as follows: Section 2 describes the time-of-flight camera/sensor technology, Section 3 presents the structure and motion model constraints for planar feature, Section 4 describes formulation of spatio-temporal RANSAC algorithm, Section 5 describes few applications of the framework and Section 6 presents experimental results and discussion, followed by conclusion in Section 7 .

\section{Time-of-flight camera}

Time-of-flight (TOF) sensors estimate distance to a target using the time-of-flight of a modulated infrared (IR) wave between the sender and the receiver. The sensor illuminates the scene with a modulated infrared waveform that is reflected back by the object. A CMOS (complementary metal-oxide-semiconductor) based lock in CCD (charge-coupled device) sensor samples four times per period. Each of these $(64 \times 48)$ smart pixels, known as Photonic Mixer Devices (PMD) [64], measure four samples $a_{0}, a_{1}, a_{2}, a_{3}$ at quarter wavelength intervals. The phase $\varphi$ of the reflected wave is computed by $[65,29]$

$\varphi=\arctan \frac{a_{0}-a_{2}}{a_{1}-a_{3}}$.

The amplitude $A$ (of reflected IR light) and the intensity $B$ representing the gray scale image returned by the sensor are respectively given by

$A=\frac{\sqrt{\left(a_{0}-a_{2}\right)^{2}+\left(a_{1}-a_{3}\right)^{2}}}{2}, \quad B=\frac{a_{0}+a_{1}+a_{2}+a_{3}}{4}$.

With measured phase $\varphi$, known modulation frequency $f_{\text {mod }}$ and precise knowledge of speed of light $c$, it is possible to measure the un-ambiguous distance $r$ from the camera,

$r=\frac{c \cdot \varphi}{4 \pi f_{\bmod }}$.

With a modulation wavelength of $\lambda_{\text {mod }}$, this leads to a maximum possible unambiguous range of $\left(\lambda_{\bmod } / 2\right)$. For a typical camera such as PMD $3 \mathrm{k}-\mathrm{S}, f_{\text {mod }}=20 \mathrm{MHz}$ and with a speed of light $c$ given by $3 \times 10^{8} \mathrm{~m} / \mathrm{s}$, the non-ambiguous range $r_{\max }$ of the TOF camera is given as

$r_{\max }=\frac{c}{2 f_{\bmod }}=\frac{3 \times 10^{8}}{2 \cdot 20 \times 10^{6}}=7.5 \mathrm{~m}$. 


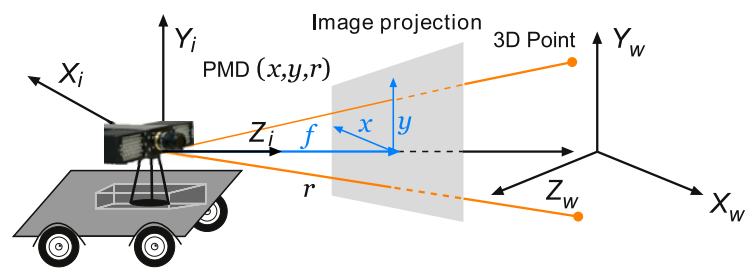

Fig. 1. Time-of-flight sensor geometry with mobile platform.

The sensor returns a range $r$ value for each pixel as a function of pixel coordinates $(x, y)$ as shown in Fig. 1 .

The range values are used to compute $3 \mathrm{D}$ position $\boldsymbol{X}(X, Y, Z)$ of the point

$Z=r(x, y) \cdot \frac{f}{\sqrt{f^{2}+x^{2}+y^{2}}} ; \quad X=Z_{\frac{f}{f}}^{x} ; \quad Y=Z \frac{y}{f}$,

where $f$ is the focal length of the camera.

\section{Structure and motion constraints}

In the following section we will discuss the motion model and the planar feature parameters essential to derive the spatiotemporal RANSAC formulation for a planar feature.

\subsection{Motion model}

Consider a TOF camera moving in space. Let $\{i\}$ denote the frame of reference at time stamp $i, 1 \leq i \leq n$, attached to the camera. Let $\{W\}$ denote the fixed world reference frame. The rigid body transformation

${ }_{i}^{W} \mathcal{M}: \mathbb{R}^{3} \rightarrow \mathbb{R}^{3} ; \quad \boldsymbol{X}_{i} \mapsto \boldsymbol{X}_{W}:={ }_{i}^{W} R \boldsymbol{X}_{i}+{ }^{W} T_{i}$

is defined as the coordinate mapping from frame $\{i\}$ to world frame $\{W\}$ with rotation $\left({ }_{i}^{W} R\right)$ and translation $\left({ }^{W} T_{i}\right)$ respectively. ${ }^{1}$ Let $\overline{\boldsymbol{X}} \in \mathbb{R}^{d+1}$ denote the homogeneous coordinates of $\boldsymbol{X} \in \mathbb{R}^{3}$, then the transformation ( 3 ) in matrix form is given $b^{2}$

${ }_{i}^{W} \overline{\mathcal{M}}: \mathbb{R}^{d+1} \rightarrow \mathbb{R}^{d+1}$;

$\overline{\boldsymbol{X}}_{W}=\left[\begin{array}{c}\boldsymbol{X}_{W} \\ 1\end{array}\right]=\left[\begin{array}{cc}{ }_{i} R & { }^{W} T_{i} \\ 0 & 1\end{array}\right]\left[\begin{array}{c}\boldsymbol{X}_{i} \\ 1\end{array}\right]={ }_{i}^{W} \overline{\mathcal{M}} \overline{\boldsymbol{X}}_{i}$.

Let ${ }_{j}^{i} \overline{\mathcal{M}} \in \mathbb{R}^{4 \times 4}$ be the rigid body mapping from frame $\{j\}$ to frame $\{i\}$ then,

${ }_{j}^{i} \overline{\mathcal{M}}={ }_{W}^{i} \overline{\mathcal{M}} \quad{ }_{j}^{W} \overline{\mathcal{M}}=\left({ }_{i}^{W} \overline{\mathcal{M}}\right)^{-1 W}{ }_{j}^{W} \overline{\mathcal{M}}$.

Hence

${ }_{j}^{i} \overline{\mathcal{M}}=\left[\begin{array}{cc}\left({ }_{i}^{W} R^{\top}\right)\left({ }_{j}^{W} R\right) & \left({ }_{i}^{W} R^{\top}\right)\left({ }^{W} T_{j}-{ }^{W} T_{i}\right) \\ 0 & 1\end{array}\right]$.

\subsection{Equation of planar feature with linear motion}

Let $P$ be a $2 \mathrm{D}$ planar feature that is stationary during the video sequence considered. Let $\eta_{i} \in\{i\}$ be the normal vector to $P$ in frame $\{i\}$, then $\eta_{i}$ is a direction that transforms between frames of reference as

$\eta_{i}={ }_{i}^{j} R^{\top} \eta_{j}={ }_{j}^{i} R \eta_{j}$

The homogeneous coordinates of a direction (free vector) such as $\eta_{i}$ are given by

$\bar{\eta}_{i}=\left[\begin{array}{c}\eta_{i} \\ 0\end{array}\right] \in \mathbb{R}^{d+1}, \quad{ }_{i}^{j} \overline{\mathcal{M}} \bar{\eta}_{i}={ }_{i}^{j} \overline{\mathcal{M}}\left[\begin{array}{c}\eta_{i} \\ 0\end{array}\right]=\left[\begin{array}{c}{ }_{i}^{j} R \eta_{i} \\ 0\end{array}\right]=\bar{\eta}_{j}$.

\footnotetext{
1 Notations common in rigid body transformation and orientation [66].

$2 d$ is the dimension of the space. Homogeneous 4-tuples representing points in $\mathbb{R}^{3}$.
}

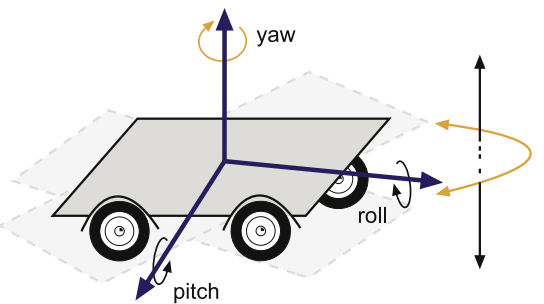

Fig. 2. Roll, pitch and dominant yaw motion.

Let $\boldsymbol{X}_{i}, \boldsymbol{X}_{j} \in P$ be different elements of the planar feature $P$ observed in different frames $\{i\}$ and $\{j\}$. Note that $\boldsymbol{X}_{i} \neq{ }_{j}^{i} \mathcal{M} \boldsymbol{X}_{j}$ in general as the points do not correspond to the same physical point in the plane, however, $\left(\boldsymbol{X}_{i},{ }_{j}^{i} \mathcal{M} \boldsymbol{X}_{j}\right)$ must both lie in $P$ in $\{i\}$. Since $\eta_{i}$ is a normal to $P$ in $\{i\}$, one has

$\left\langle\left(\overline{\boldsymbol{X}}_{i}-{ }_{j}^{i} \mathcal{M} \overline{\boldsymbol{X}}_{j}\right), \bar{\eta}_{i}\right\rangle=0$.

Thus

$\left\langle\left[\begin{array}{c}\boldsymbol{X}_{i} \\ 1\end{array}\right]-\left[\begin{array}{c}\left({ }_{i}^{W} R^{\top}\right)\left({ }_{j}^{W} R\right) \boldsymbol{X}_{j}+\left({ }_{i}^{W} R^{\top}\right)\left({ }^{W} T_{j}-{ }^{W} T_{i}\right) \\ 1\end{array}\right]\right.$,

$$
\begin{aligned}
& \left.\left[\begin{array}{c}
\eta_{i} \\
0
\end{array}\right]\right\rangle=0 \\
& \left\langle\boldsymbol{X}_{i}-\left({ }_{i}^{W} R^{\top}\right)\left({ }_{j}^{W} R\right) \boldsymbol{X}_{j}-\left({ }_{i}^{W} R^{\top}\right)\left({ }^{W} T_{j}-{ }^{W} T_{i}\right), \eta_{i}\right\rangle=0 \\
& \left\langle\boldsymbol{X}_{i}-\left({ }_{i}^{W} R^{\top}\right)\left({ }_{j}^{W} R\right) \boldsymbol{X}_{j}, \eta_{i}\right\rangle-\left\langle\left({ }_{i}^{W} R^{\top}\right)\left({ }^{W} T_{j}-{ }^{W} T_{i}\right), \eta_{i}\right\rangle=0 \\
& \left\langle\boldsymbol{X}_{i}-\boldsymbol{X}_{j},\left({ }_{j}^{W} R\right)\left({ }_{i}^{W} R^{\top}\right) \eta_{i}\right\rangle-\left\langle\left({ }^{W} T_{j}-{ }^{W} T_{i}\right),\left({ }_{i}^{W} R\right) \eta_{i}\right\rangle=0 .
\end{aligned}
$$

Let $V \in\{W\}$ denote the linear velocity then the rigid body dynamics for a moving body (an automotive) is modeled by

$\dot{T}=V ; \quad T(0)=T_{1}$

$\dot{R}=\widehat{\omega} R ; \quad R(0)=R_{1}$,

where $\omega \in\{W\}$ is the angular velocity and $\widehat{\omega} \in \mathbb{R}^{3 \times 3}$ denote the skew symmetric matrix that corresponds to vector cross product operation in 3D.

Assumption. We assume that the angular velocity $\omega$ of the camera is parallel to $\eta \in\{W\}$, the normal to the ground plane at all times and the translation velocity $V$ in the direction normal to the ground plane is constant such that

$\eta \times \omega=0$ and $\langle V, \eta\rangle=$ constant,

where $\times$ represents a cross product between two vectors. For normal motion of a vehicle, roll and pitch rotations are negligible compared to yaw motion associated with angular velocity of the turning vehicle [67], and corresponds to the common groundplane constraint (GPC) [68] (see Fig. 2). In real environments for motion captured at nearly video frame rate, the piecewise linear velocity along the normal direction can be assumed constant as evident from the experiments in Section 6. This is to be expected in the case where the camera is attached to a vehicle that moves on a plane $P$, precisely the case for the automotive example considered. In practice, the vertical degree of motion is important to model situations where the vehicle suspension is active. It is also used to identify non-ground features that the vehicle may be approaching with constant velocity.

As a consequence of (12)

$\omega=s(t) \eta \in\{W\} ; s: \mathbb{R} \rightarrow \mathbb{R}$ in time $t$.

Following (13) one can re-write (11) as

$\dot{R}=s(t) \widehat{\eta} R ; \quad R(0)=R_{1}$. 
Therefore the continuous rotation motion $R(t): \mathbb{R} \rightarrow S O(3)$ for the automobile trajectory is expressed as

$R(t)=\exp \left(\theta\left(t_{i}\right) \widehat{\eta}\right) R_{1} ; \quad \theta\left(t_{i}\right)=\int_{0}^{t_{i}} s(\tau) d \tau$

where time at frame $\{i\}$ is $t_{i}$ and

${ }_{i}^{W} R=R\left(t_{i}\right)$.

By definition ${ }_{i}^{W} R \eta_{i}=\eta$ and hence,

$$
\begin{aligned}
\eta_{i} & ={ }_{i}^{W} R^{\top} \eta \\
& =R_{1}^{\top} \exp \left(\theta\left(t_{i}\right) \widehat{\eta}\right)^{\top} \eta \\
& =R_{1}^{\top} \eta=\eta_{1} .
\end{aligned}
$$

Using (15), we can re-write (10) as

$\left\langle\boldsymbol{X}_{i}-\boldsymbol{X}_{j}, \eta_{1}\right\rangle-\left\langle{ }^{W} T_{j}-{ }^{W} T_{i}, \eta_{1}\right\rangle=0$.

We assume the frames are taken at constant time interval $\delta t$ and hence $t_{i}=\delta t(i-1)+t_{1}$. Since $\langle V, \eta\rangle$ is constant and $t_{1}=0$, the linear translation motion ${ }^{W} T_{i}$ satisfies

$\left\langle{ }^{W} T_{i}, \eta_{i}\right\rangle=\langle V, \eta\rangle \delta t(i-1)+\left\langle T_{1}, \eta_{1}\right\rangle$.

Using assumption (12), define $\alpha \in \mathbb{R}$ to be

$\alpha=\langle V, \eta\rangle \delta t=$ constant.

Thus, from (16) and (17), the structure and motion constraint that $\boldsymbol{X}_{i}, \boldsymbol{X}_{j}$ lie in the plane $P$ can be expressed as

$$
\left\langle\boldsymbol{X}_{i}-\boldsymbol{X}_{j}, \eta_{1}\right\rangle-\alpha(j-i)=0 \text {. }
$$

This is an equation for a plane $P$ parameterized by $\eta_{1} \in S^{2}\left(\left\|\eta_{1}\right\|\right.$ $=1$ ) and motion parameter $\alpha \in \mathbb{R}$. An additional parameter, the distance $h \in \mathbb{R}$ of the plane $P$ from the origin in frame $\{1\}$ in the direction $\eta_{1}$, completes the structure and motion constraints of a planar feature. Note that $\alpha$ is the component of translational camera velocity in the direction normal to the planar feature $P$. The component $\alpha$ will be the defining parameter for the temporal component of the $3 \mathrm{D}$ planar feature that is identified in the RANSAC algorithm (see Section 4).

Let $\overline{\overline{\boldsymbol{X}}}_{i}$ be a $4 \mathrm{D}$ spatio-temporal coordinate that incorporates both spatial coordinates $\boldsymbol{X}_{i}$ and a reference to the frame index or time coordinates $i$

$\overline{\overline{\boldsymbol{X}}}_{i}=\left[\begin{array}{c}\boldsymbol{X}_{i} \\ i\end{array}\right]$.

Associated with this we define a normal vector that incorporates the spatial normal direction $\eta_{1}$ and the motion parameter $\alpha$

$\overline{\bar{\eta}}=\left[\begin{array}{c}\eta_{1} \\ \alpha\end{array}\right]$.

Using these definitions (19) may be re-written as

$\left\langle\overline{\overline{\boldsymbol{X}}}_{i}-\overline{\overline{\boldsymbol{X}}}_{j}, \overline{\bar{\eta}}\right\rangle=0$.

\section{Spatio-temporal RANSAC algorithm}

In this section we present the spatio-temporal RANSAC algorithm and compute a 3D spatio-temporal planar hypothesis based on the structure and motion model derived in Section 3.2 and a minimal data set.

\subsection{Computing a spatio-temporal planar hypothesis}

Eq. (19) provides a constraint that $\left(\overline{\overline{\boldsymbol{X}}}_{i}-\overline{\overline{\boldsymbol{X}}}_{j}\right) \in \mathbb{R}^{4}$ lies in the 3D spatio-temporal planar feature $P$ in $\mathbb{R}^{4}$ with parameters $\eta_{1} \in S^{2}$, $\alpha \in \mathbb{R}$ and $h \in \mathbb{R}$. Given a sample of four points $\left\{\overline{\overline{\boldsymbol{X}}}_{i_{1}}, \overline{\overline{\boldsymbol{X}}}_{i_{2}}, \overline{\overline{\boldsymbol{X}}}_{i_{3}}, \overline{\overline{\boldsymbol{X}}}_{i_{4}}\right\}$, one can construct a normal vector $\overline{\bar{\eta}}$ to $P$ by taking the 4D cross product [69] (see the Appendix)

$\overline{\bar{\eta}}_{o}=\operatorname{cross}_{4}\left(\overline{\overline{\boldsymbol{X}}}_{i_{1}}-\overline{\overline{\boldsymbol{X}}}_{i_{2}}, \overline{\overline{\boldsymbol{X}}}_{i_{1}}-\overline{\overline{\boldsymbol{X}}}_{i_{3}}, \overline{\overline{\boldsymbol{X}}}_{i_{1}}-\overline{\overline{\boldsymbol{X}}}_{i_{4}}\right) \in \mathbb{R}^{4}$,

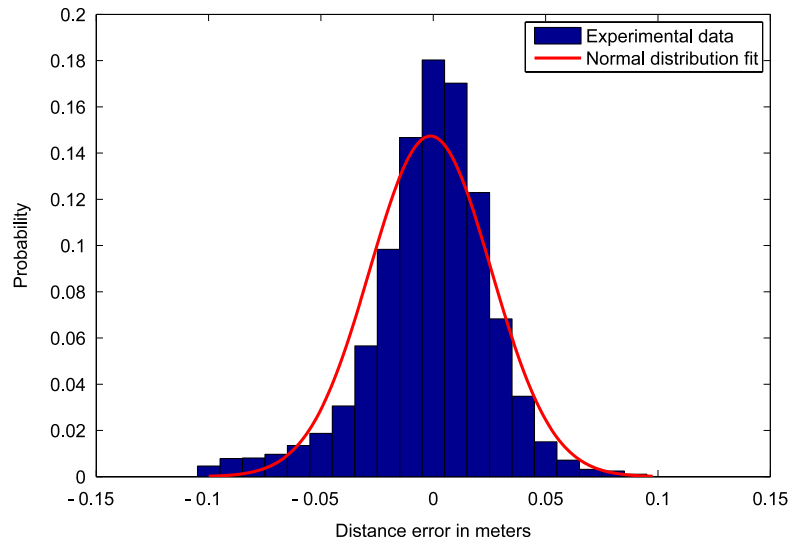

Fig. 3. Statistical distribution of data points around a planar feature derived from experimental data documented in Section 6. Normal distribution fit is obtained through maximum likelihood estimate $\mathcal{N}(0.001,0.02)$. The graph shows that the noise in range data obtained through TOF camera is approximately Gaussian.

where $\overline{\overline{\boldsymbol{X}}}_{i} \in\{\{1\}, \ldots,\{n\}\}$. To apply the constraint $\eta_{1} \in S^{2}$ we normalize $\overline{\bar{\eta}}_{o}=\left(\overline{\bar{\eta}}_{o}^{x}, \overline{\bar{\eta}}_{o}^{y}, \overline{\bar{\eta}}_{o}^{z}, \overline{\bar{\eta}}_{o}^{t}\right)$ by

$\overline{\bar{\eta}}=\frac{1}{\beta} \overline{\bar{\eta}}_{o} ; \quad \beta=\sqrt{\left(\overline{\bar{\eta}}_{o}^{x}\right)^{2}+\left(\overline{\bar{\eta}}_{o}^{y}\right)^{2}+\left(\overline{\bar{\eta}}_{o}^{z}\right)^{2}}$.

The resulting estimate $\overline{\bar{\eta}}=\left(\eta_{1}, \alpha\right)$ is an estimate of the normal $\eta_{1} \in S^{2}$ and $\alpha$, the normal vector component of translation velocity (18).

Note that the depth parameter $h$ can be determined by

$h_{1}=\left\langle\boldsymbol{X}_{i}, \eta_{1}\right\rangle-\alpha(i-1)$.

However, the parameter $h$ is not required for the robust estimation phase of the RANSAC algorithm and is evaluated in the second phase where a refined model is estimated.

\subsection{Statistical distribution of $4 D$ data points}

The spatio-temporal data points that have a probability $p$ of lying in the planar feature are defined as inliers. Due to the noise in range measurements of TOF camera, the distance of these inliers from the model (planar feature) are noisy. In the present case, a Gaussian distribution is assumed with $\mathcal{N}(0, \sigma)$ as shown in Fig. 3. This assumption is equivalent to assuming sufficient signal-tonoise ratio (SNR) of the return signal [70]. As a consequence, the point square distance $a_{\perp}^{2}$,

$a_{\perp}^{2}=\left(\left\langle\left(\overline{\overline{\boldsymbol{X}}}-\overline{\overline{\boldsymbol{X}}}_{i_{1}}\right), \overline{\bar{\eta}}\right\rangle\right)^{2} ; \quad \overline{\overline{\boldsymbol{X}}} \in$ all spatio-temporal data points,

of the inliers [71] from the planar feature associated with the data point $\overline{\boldsymbol{X}}_{i}$, have a chi-squared distribution $\chi^{2}$. Since we consider a spatio-temporal planar feature, there are three degrees of freedom in the chi-squared distribution. Let $F_{\chi_{3}^{2}}$ denote the cumulative frequency of three degree of freedom of chi-squared distribution $\chi_{3}^{2}$ then one can define the threshold coefficient $q^{2}$ by

$q^{2}=F_{\chi_{3}^{2}}^{-1}(p) \sigma^{2}$.

Thus, the statistical test for inliers is defined by

$\begin{cases}\text { inliers } & a_{\perp}^{2}<q^{2} \\ \text { outliers } & a_{\perp}^{2} \geq q^{2} .\end{cases}$

In the experiments documented in Section 6 , we use a value of $p=0.95$. In this case the threshold is $q^{2}=7.81 \sigma^{2}$ where $\sigma$ is determined empirically. Spatial ground plane estimation algorithms using single 3D images $[45,56,57]$ are associated with two degree of chi-squared distribution since they lack temporal dimension. As a result the same analysis leads to a threshold of $q^{2}=5.99 \sigma^{2}$ (for $p=0.95$ ). The additional threshold margin 
for the proposed spatio-temporal algorithm quantifies the added robustness that comes from incorporating the temporal dimension along with the data available by incorporating multiple images from the video stream. This leads to significant improvement in robustness and performance of the proposed algorithm over single image techniques. The resulting spatio-temporal RANSAC algorithm is outlined in Algorithm 1.

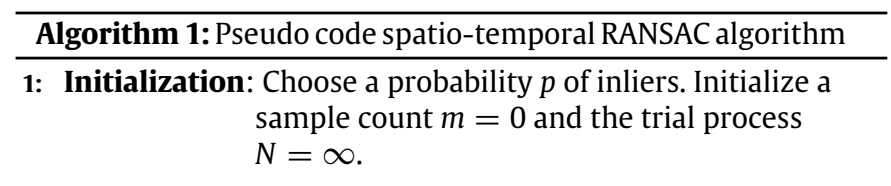

2: repeat

a. Select at random, 4 spatio-temporal points $\left(\overline{\overline{\boldsymbol{X}}}_{i_{1}}, \overline{\overline{\boldsymbol{X}}}_{i_{2}}\right.$, $\left.\overline{\overline{\boldsymbol{X}}}_{i_{3}}, \overline{\overline{\boldsymbol{X}}}_{i_{4}}\right)$.

b. Compute the temporal normal vector $\overline{\bar{\eta}}$ according to (23) and (24).

c. Evaluate the spatio-temporal constraint (22) to develop a consensus set $C_{m}$ consisting of all data points classified as inliers according to (27).

d. Update $N$ to estimate the number of trials required to have a probability $p$ so that the selected random sample of 4 points is free from outliers as,

$$
N=\log (1-p) / \log \left(1-\frac{\text { number of inliers }}{\text { number of points }}\right)^{4} \text {. }
$$

until at least $N$ trials are complete

3: Select the consensus set $C_{m}^{*}$ that has the most inliers.

4: Optimize the solution by re-estimating from all

spatio-temporal data points in $C_{m}^{*}$ by maximizing the likelihood of the function $\phi$

$$
\begin{aligned}
& \phi(\overline{\bar{\eta}}, h)=\sum_{\overline{\overline{\boldsymbol{X}}} \in C_{m}^{*}}(\langle\overline{\bar{\eta}}, \overline{\overline{\boldsymbol{X}}}\rangle-h)^{2} \\
& \left.\mathcal{L}(\phi)=\prod_{\overline{\overline{\boldsymbol{X}}} \in C_{m}^{*}} \phi(\overline{\overline{\boldsymbol{X}}} \mid \overline{\bar{\eta}}, h) ; \quad \hat{\overline{\bar{\eta}}}, \hat{h}\right)=\arg \max _{\overline{\bar{\eta}}, h}(\mathcal{L}),
\end{aligned}
$$

where we assume a normal distribution in observed depth.

\section{Applications}

The planar feature estimation algorithm in 4D is an approach that can be utilized in multiple scenarios. We have presented two potential applications of the algorithm relevant to autonomous navigation.

\subsection{Ground plane detection}

The algorithm provides an effective technique for robust planar feature estimation and detection. Real scenarios present a number of planar features such as walls and roads. The dominating planar features for autonomous ground vehicle is the ground plane. With constant normal velocity component $\alpha$, Eq. (18), it is possible to detect ground plane where the value of $\alpha$ is small. The smaller constant value of $\alpha$ represents a planar feature (ground in this case) due to the fact that piecewise linear velocity in the normal direction of the automotive motion is small and constant over the number of frames recorded at frame rate. Detection of ground plane in spatio-temporal domain provides an added advantage for cases where there is occlusion and single frame detection is not possible. Section 6.1 presents number of examples with $\alpha$ obtained for ground plane.

\subsubsection{Obstacle detection}

An obstacle detection algorithm is a natural extension once a robust estimation of planar ground surface is available. In the proposed algorithm each spatio-temporal data point is evaluated and categorized as traversable and non-traversable objects or obstacles. Traversable objects are the points that can be comfortably driven over in a vehicle. The approach taken is similar to methods proposed in $[20,72]$. The estimated Euclidean distance $\hat{d}$ to the plane for an arbitrary data point $\overline{\bar{X}}$ is defined as

$\hat{d}=\langle\overline{\overline{\boldsymbol{X}}}, \hat{\bar{\eta}}\rangle-\hat{h}$.

Objects (in each frame) are segmented from the ground plane by a threshold $\tau$ as

$\overline{\overline{\boldsymbol{X}}}= \begin{cases}\text { Obstacle } & |\hat{d}| \geq \tau_{o} \\ \text { Traversable object } & |\hat{d}|<\tau_{o},\end{cases}$

where $\tau_{0}$ is set by the user for the application under consideration. This threshold segmentation helps in reliable segregation of potential obstacles. The allowance of larger threshold in inliers for plane estimation makes obstacle detection phase robust for various applications especially for on road obstacle detection.

\subsection{Segmentation of planar features}

The 4D spatio-temporal normal vector has a spatial component and a temporal component $\alpha=V \cos \theta$ as indicative of a normal velocity component, where $\theta$ is the angle between the vehicle and the normal to the planar surface. Therefore by considering features with arbitrary $\alpha$ values one can segment the scene in the velocity domain. Using a low number of frames in the spatio-temporal RANSAC algorithm the vehicle motion constraints become less restrictive in rejecting potential planar features and the algorithm (run in multiple feature mode) will identify all planar features in the scene and provide estimates of the normal velocity $\alpha$ of the vehicle to each feature. Suitable values of $\alpha$ and structure parameters for the planar feature can be used to identify potential hazards for the moving vehicle and a braking mechanism can be initiated in an autonomous vehicle. A possible criterion for identification of a dangerous planar feature would be

hazardous planar surface/feature $=\alpha>\tau_{s}$,

where $\tau_{s}$ is a threshold selected according to application/scenario. The experiment in Section 6.2 applied this method to a scenario involving the test vehicle (fitted with PMD TOF camera) approaching a wall.

\section{Experimental results and discussions}

Experiments were performed using real video data recorded from PMD 3k-S TOF camera mounted on a vehicle with an angle varying between $2^{\circ}$ and $20^{\circ}$ to the ground. The camera records at approximately $20 \mathrm{fps}$ and provides both gray scale and range images in real time. The sensor has a field of view of $33.4^{\circ} \times$ $43.6^{\circ}$. The video sequences depict scenarios in an under cover car park due to limitations of the available hardware in dealing with Australian full sunlight conditions. ${ }^{3}$ PMDTech claims that current TOF sensors perform equally well in bright day light [73].

\footnotetext{
3 While the paper proposed an algorithm using range data from time-of-flight (TOF) cameras in general; experimental verification was undertaken using an earlier version of PMD 3k-S TOF camera. The specific prototype does not work in outside conditions and was not calibrated to use Suppression Background Illumination module (SBI). Since, the proposed algorithm is intensity independent (uses range data only), the framework is equally applicable to newer models of TOF cameras that work in outside conditions.
} 
a

b
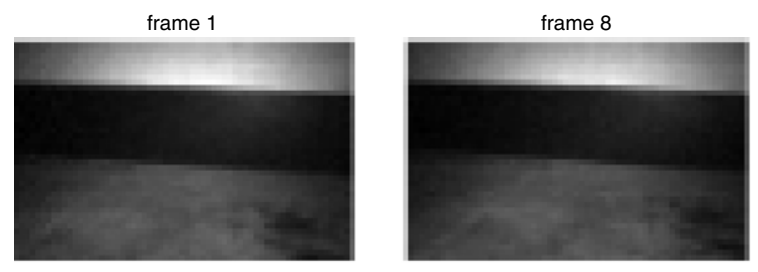

d

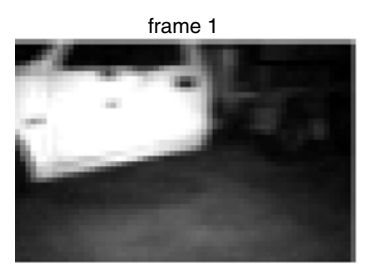

9

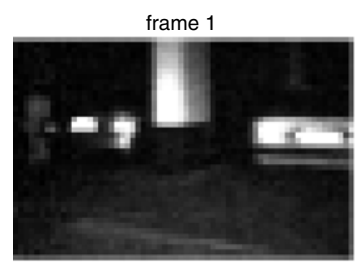

j

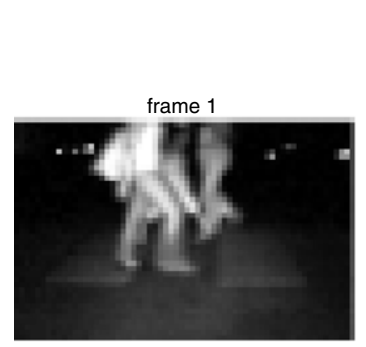

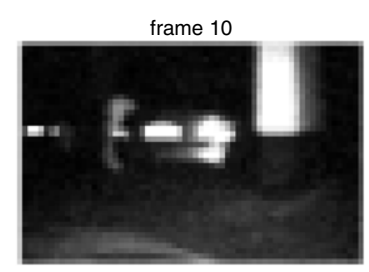

e

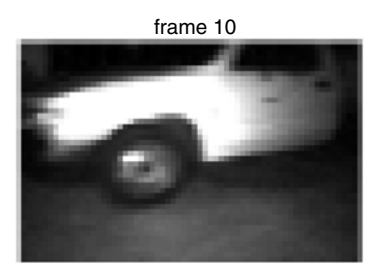

$\mathrm{h}$

$\mathrm{k}$

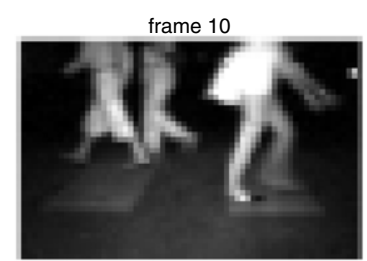

C

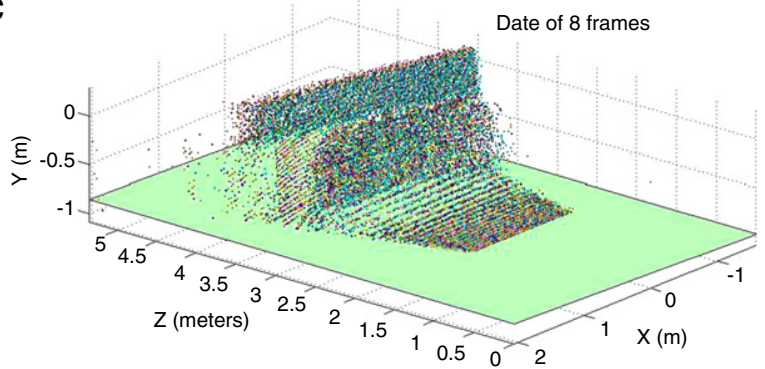

f
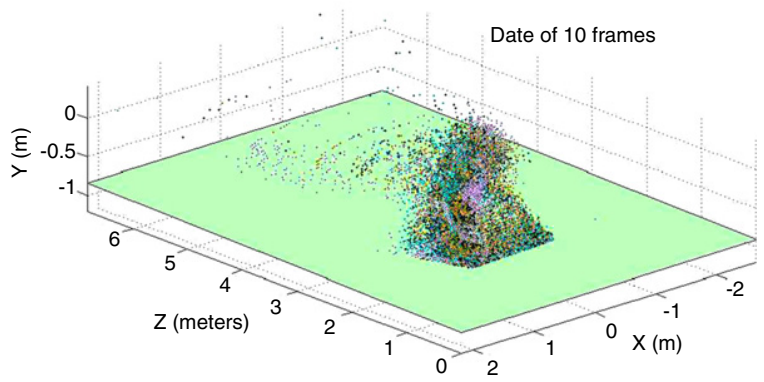

i

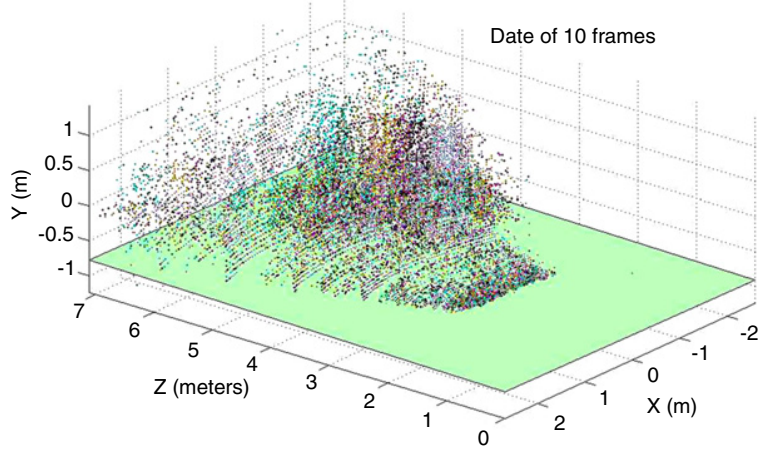

I

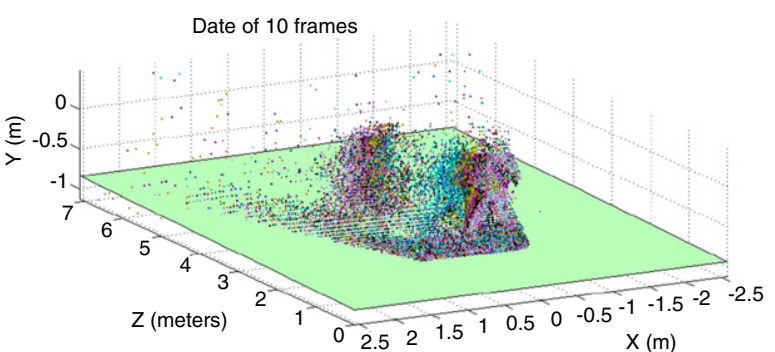

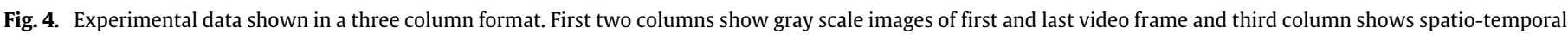

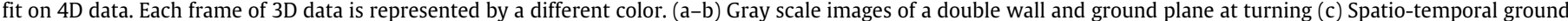

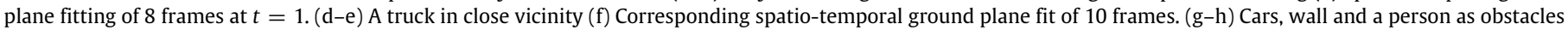
at turning. (i) Corresponding spatio-temporal ground plane fit. (j-k) Pedestrians. (l) Ground plane fit.

In particular, we consider cases with pedestrians, close by vehicles, obstacles, curbs/footpaths and walls etc. Seven experimental scenarios have been presented to evaluate the robustness of the algorithm against real objects and also compared with standard 3D RANSAC algorithm.

\subsection{Planar feature (ground plane) detection}

In the following experiments the gray scale images, when shown, represent the first and the last frame of video data. It is not possible to have a $4 \mathrm{D}$ visualization environment, therefore a 3D multi-frame representation (each data frame represented in different color) is used to present spatio-temporal video range data. The estimated spatio-temporal planar feature is represented in frame $\{1\}$. The final solution is rotated for better visualization. In the first set of experiments shown in Fig. 4 and Table 1 (sequences
1-4), four different scenarios are presented. The first scenario shows multiple walls at varying levels of depth and a ground plane. The algorithm correctly picks the ground plane rejecting other planar features. In the next scenario, a truck in close vicinity is obstructing the clear view but the ground plane has been identified by exploiting the full video sequence of the data. A number of obstacles including cars, wall and a person are visible while the car is maneuvering a turn in the third scenario. The algorithm clearly estimates actual ground plane. In the fourth scenario the result is not perturbed by passing pedestrians and the algorithm robustly identifies the ground plane.

In a typical sequence; a 8-10 frame data is enough to resolve a ground plane even in the presence of some kind of occlusion. This is demonstrated in Fig. 5 (sequence 5 with 2-21 frames data) where we consider running the algorithm separately starting with 
Table 1

Experimental data for ground plane estimation ${ }^{\mathrm{a}}$.

\begin{tabular}{|c|c|c|c|c|}
\hline Sequence no & Sequence & No of frames used & $|\alpha|$ (m/frame) & Camera angle to ground $\left({ }^{\circ}\right)$ \\
\hline 1 & Double wall & 8 & 0.0016 & 12 \\
\hline 2 & Moving truck & 10 & 0.0017 & 13 \\
\hline 3 & Multiple objects & 10 & 0.0021 & 3 \\
\hline 4 & Pedestrian & 10 & 0.0020 & 12 \\
\hline 5 & Front wall & 10 & 0.0018 & 21 \\
\hline 6 & Speed bump 1 & 10 & 0.0040 & 11 \\
\hline 7 & Speed bump 2 & 10 & 0.0035 & 13 \\
\hline
\end{tabular}

${ }^{\text {a }}$ Few video sequences provided with submission.

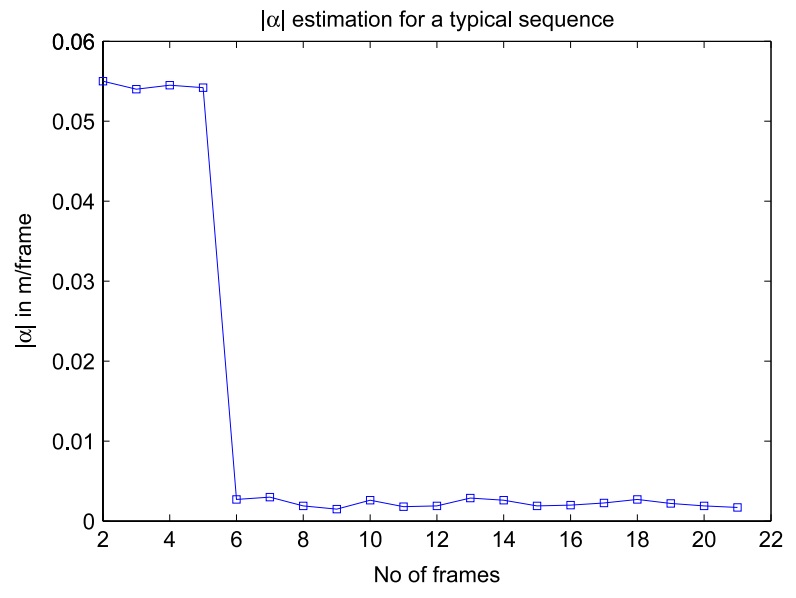

Fig. 5. Normal velocity component $|\alpha|$ of $4 \mathrm{D}$ spatio-temporal RANSAC fit for a number of frames (sequence 5).

2 frames up to 21 frames in cases where there is an occlusion. The dominant occlusion is clearly picked for low frame choices 2-5 frames with an associated $\alpha \neq 0$. The spatio-temporal RANSAC algorithm rejects the wall and picks the ground plane for higher number of frames ( 10 frames).

In another set of experiments using sequences 6 and 7 (shown in Figs. 6-9), frames are captured while the vehicle passes over the speed bumps to evaluate the robustness of the algorithm. Uneven road surface increases noise in the horizontal plane as shown in Fig. 7(c) and Fig. 9(c). The sequences shown in Figs. 6-7 illustrate that even if the vehicle moves over an un-even plane that results in change of vehicle pitch angle (in this case the dominant noise component), the algorithm is robust enough in estimating the ground plane.

In another experiment shown in Fig. 10(a) (sequence 5 with single frame data), the standard RANSAC algorithm is applied using a single frame data for comparison. The obvious failure of a standard RANSAC algorithm is due to the bias of planar data points toward the wall. On the other hand, the proposed algorithm has correctly identified the ground surface in Fig. 10(b) by simply incorporating more frames (10 frames and $|\alpha|=0.0018$ ) due to the availability of temporal data without imposing any scene constraint.

\subsubsection{Obstacle detection}

The proposed obstacle detection algorithm is applied after robust estimation of ground plane. In the experiment shown in Fig. 11, pedestrians are segmented with $\tau_{0}=0.1$ by the obstacle detection algorithm after correct identification of ground plane. This threshold implies that objects with a height greater than $10 \mathrm{~cm}$ (shown in red color) are considered as obstacle where data points close to ground plane are ignored (traversable objects) with this threshold. The graph shows the statistical and threshold representation of the data. This application of the algorithm is clearly one of its most important outcome of this approach.
Table 2

$\alpha$ and normal velocity in experiments.

\begin{tabular}{lll}
\hline Sequence no & $\alpha(\mathrm{m} /$ frame $)$ & Normal velocity $(V)$ \\
\hline 4 & 0.0025 & $0.0500 \mathrm{~m} / \mathrm{s}=0.1800 \mathrm{~km} / \mathrm{h}$ \\
5 & $0.0013($ at $3.06 \mathrm{~m})$ & $0.0260 \mathrm{~m} / \mathrm{s}=0.0936 \mathrm{~km} / \mathrm{h}$ \\
5 & $0.0810($ at $2.15 \mathrm{~m})$ & $1.6200 \mathrm{~m} / \mathrm{s}=5.8320 \mathrm{~km} / \mathrm{h}$ \\
\hline
\end{tabular}

The experimental results are straightforward and show excellent performance.

\subsection{Segmentation}

The magnitude and direction of the normal velocity is an important aspect of the spatio-temporal RANSAC. An effective segmentation of planar features is achieved with relatively small number of frames (2-3 frames). Two frames of range data are sufficient for the estimation of instantaneous velocity. Instead an average effect by incorporating 3 frames is proposed based on the fact that range data is noisy in general. In Fig. 13 the normal velocity $(\alpha)$ is shown as the car decelerates while approaching toward the wall. A positive $\alpha$ indicates a car approaching an obstacle and vice versa for the axes considered. In Fig. 13, the low value of $\alpha$ in the right hand of the graph indicates where the wall obstacle is not visible to the camera and the ground plane is consistently picked. This abruptly changes at $2.15 \mathrm{~m}$ where the wall obstacle becomes dominant in the scene (see Table 2 ). The associated values of $\alpha$ represent the normal velocity of the vehicle to the wall. The deceleration of the vehicle as it approaches the wall is clear in the values of $\alpha$. In the normal velocity domain, ground plane has two characteristics. First, $\alpha$ needs to be constant and second, it needs to be small to adhere to the movement of automobile on the ground plane. Since $\alpha$ is representative of the normal velocity of the feature, $\alpha>\tau_{s}$ shown in Fig. 13 is used to segment the scene into different $\alpha$ values such as wall and a ground plane.

In another set of experiments, different planar surfaces were segmented using the proposed algorithm. Planar surfaces are segmented sequentially from the $4 \mathrm{D}$ data set. The inliers which are associated with first planar surface are eliminated for the estimation of the second planar surface. The algorithm can be stopped when either the desired number of planar features have been extracted or the whole data set has been exhausted and segmented into planar surfaces. In Fig. 12, the algorithm is applied on two different sequences. In the first sequence, two major planar features (road and wall) are segmented. In the second sequence, three different planar surfaces are segmented sequentially from the spatio-temporal range data. Table 3 shows number of inliers associated with each planar surface, segmented through the proposed algorithm.

\subsection{Performance}

The proposed 4D spatio-temporal RANSAC algorithm's computation cost is associated with picking the normal vector to the 3D planar feature by random sampling. This eliminates any 

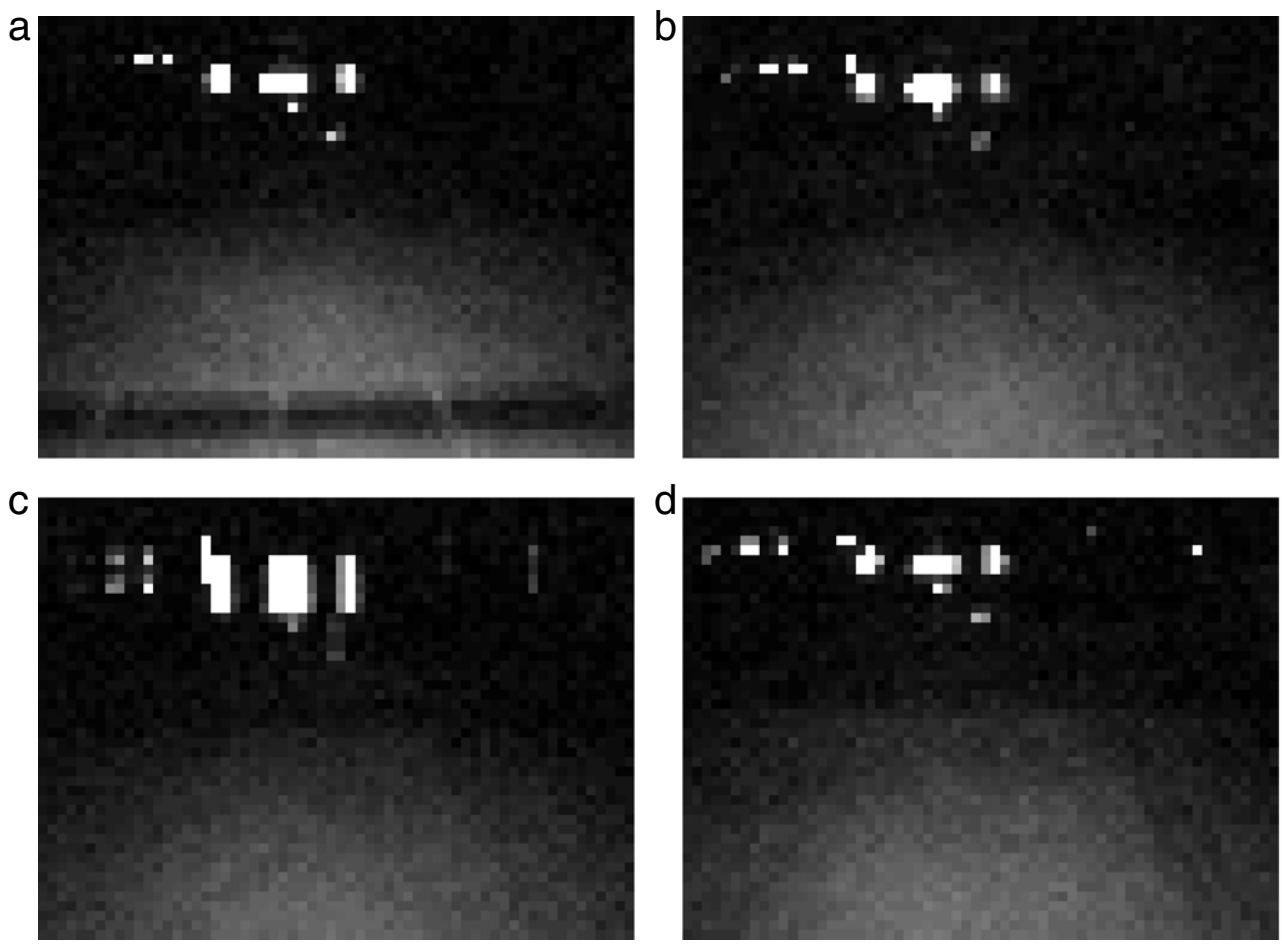

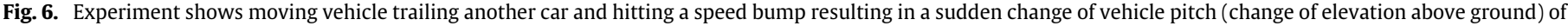

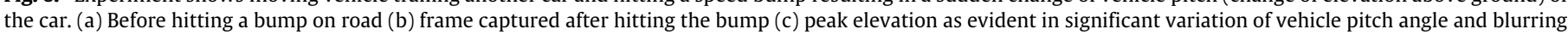
of tail lights of car in front is captured by the camera (d) frame captured after the bump and vehicle movement is stabilized.

a

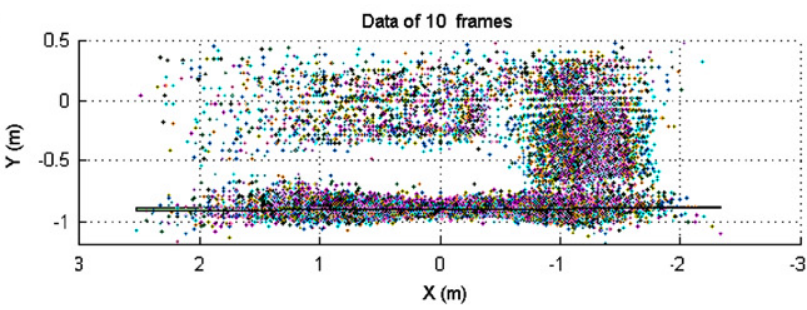

C

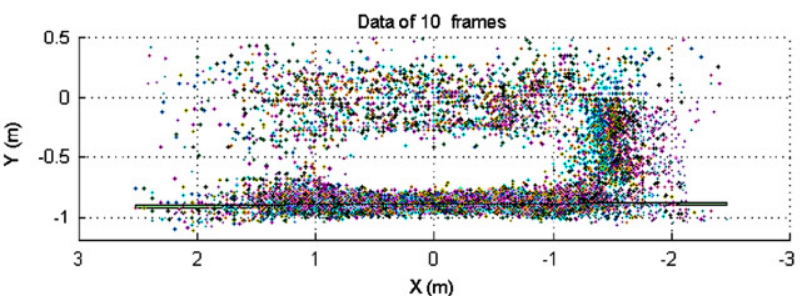

e

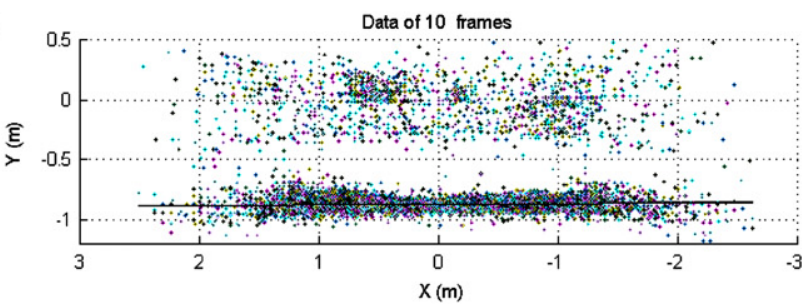

b

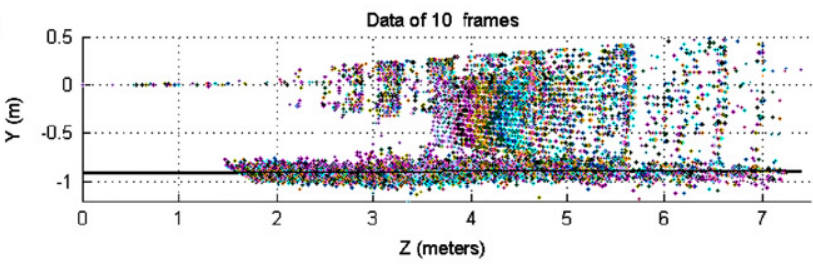

d

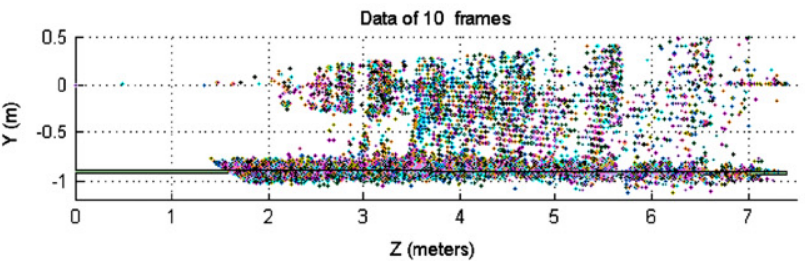

f

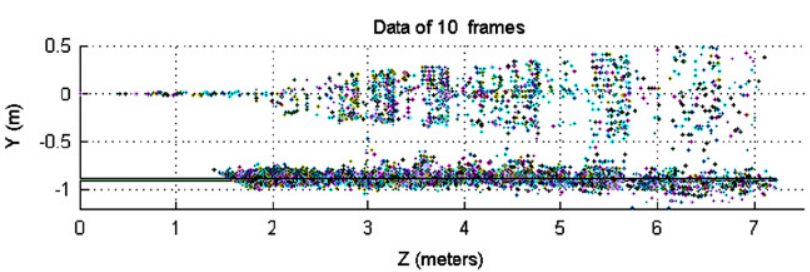

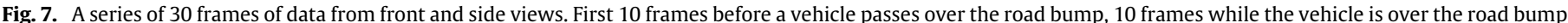

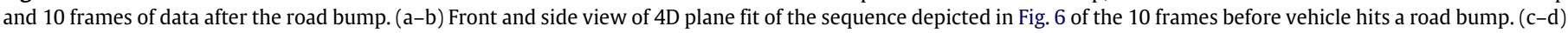

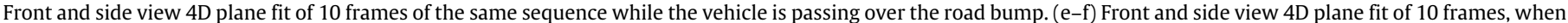
the vehicle has passed over the road bump.

computation cost associated with pre-processing images, and in particular there is no requirement for image registration or feature matching, resulting in considerable computational efficiency with respect to conventional algorithms. 
a

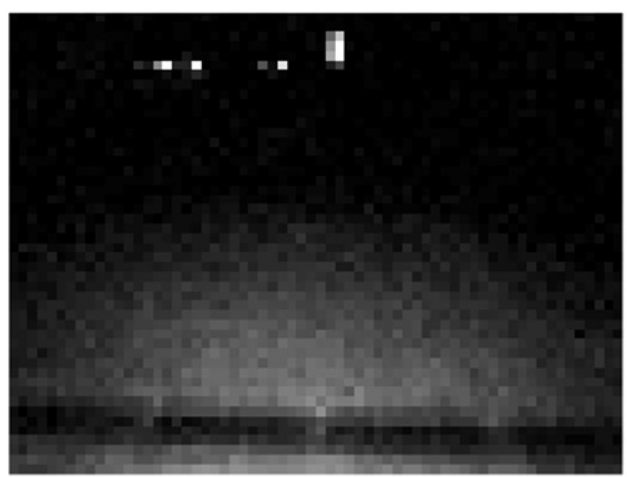

C

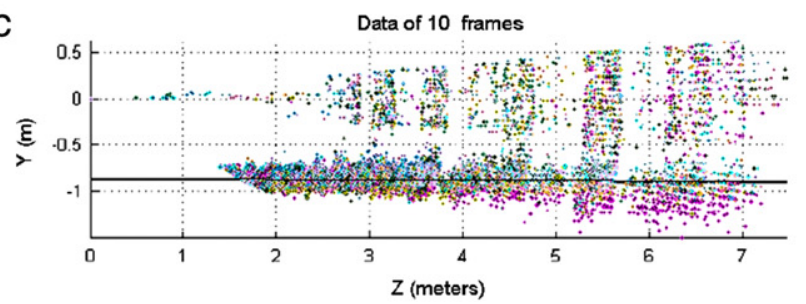

b

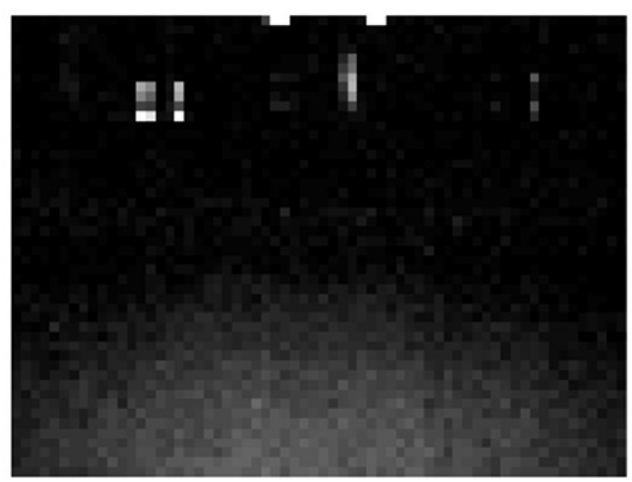

d

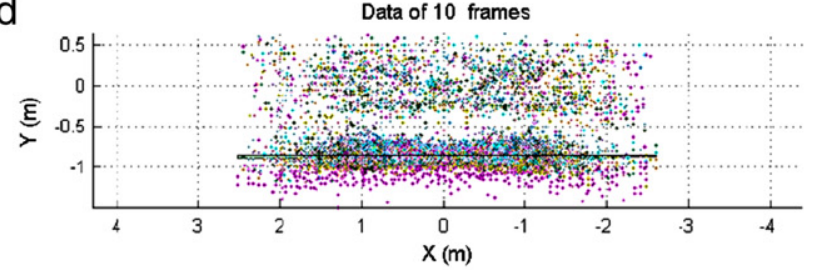

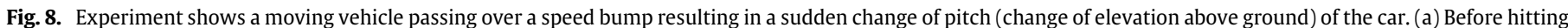

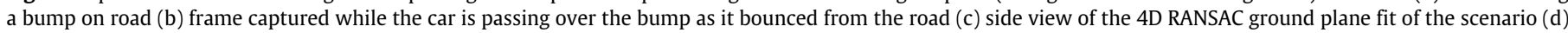
frontal view of the spatio-temporal ground plane fit of the same scenario.

a

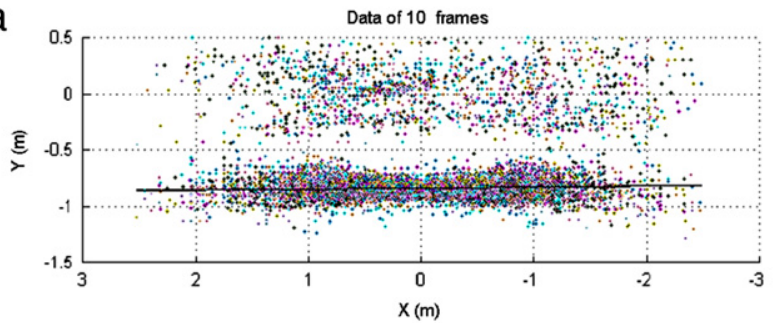

C

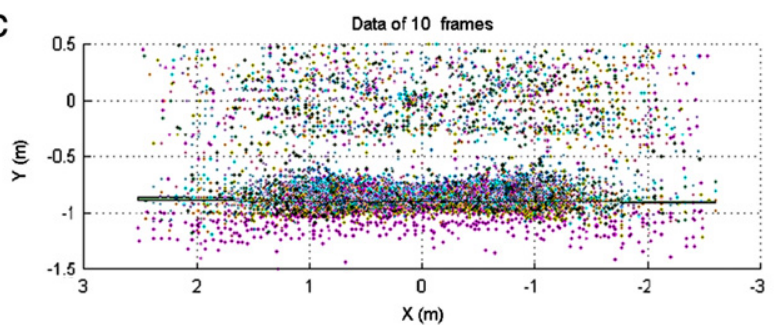

e

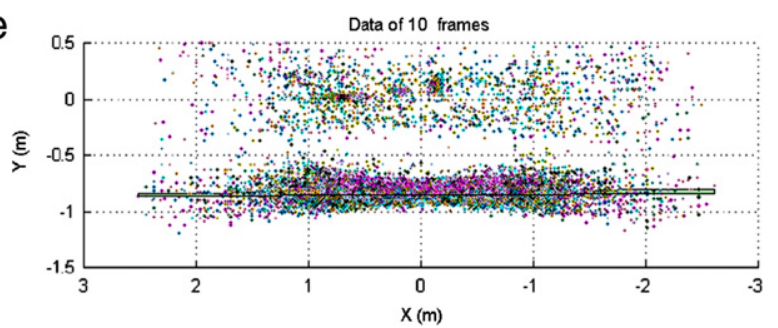

b

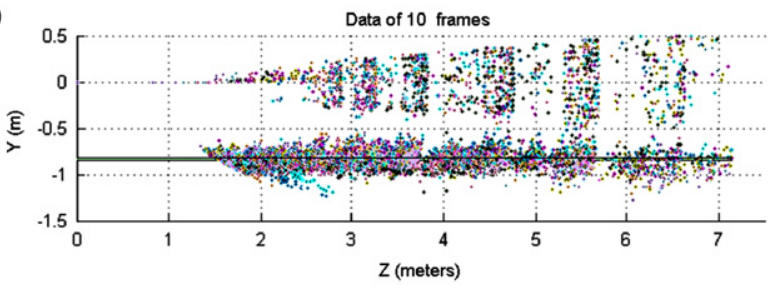

d

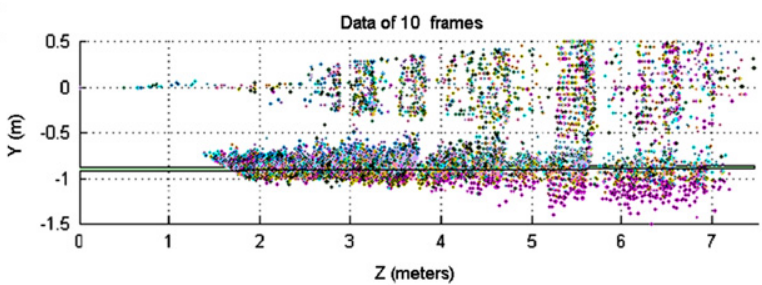

f

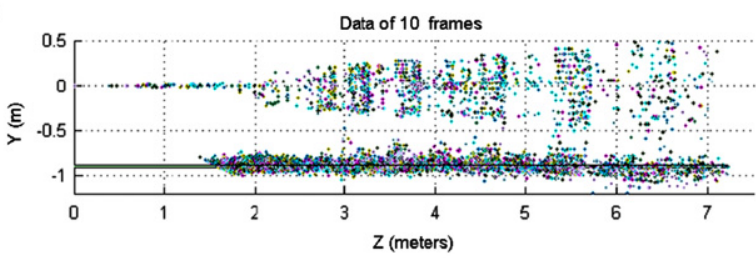

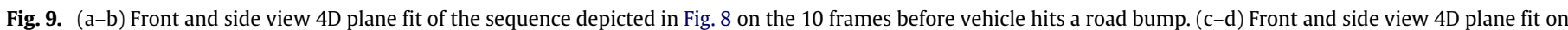

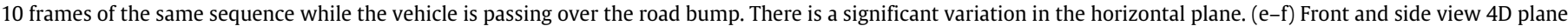
fit on 10 frames, when the vehicle has passed over the road bump.

The experiments were performed on a PC machine with Intel Core 2 Duo $3 \mathrm{GHz}$ processor and 2 GB RAM. The algorithm is implemented in MATLAB. The computation cost varies with the number of inliers and the planar surface occlusion in the range data as shown in Fig. 14. For a typical example using five frames for $4 \mathrm{D}$ data for sequence 4 (pedestrian crossing), the algorithm picks 7461 inliers out of a data set of $15360,4 \mathrm{D}$ data points. Note that a single 3D frame consists of $48 \times 64=3072$ data points. Hence a five 
a

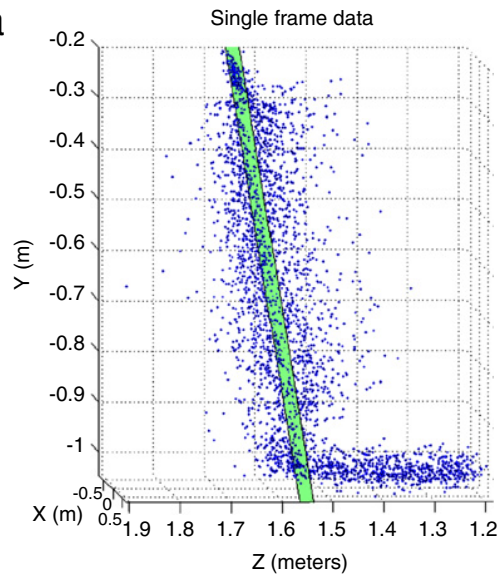

b

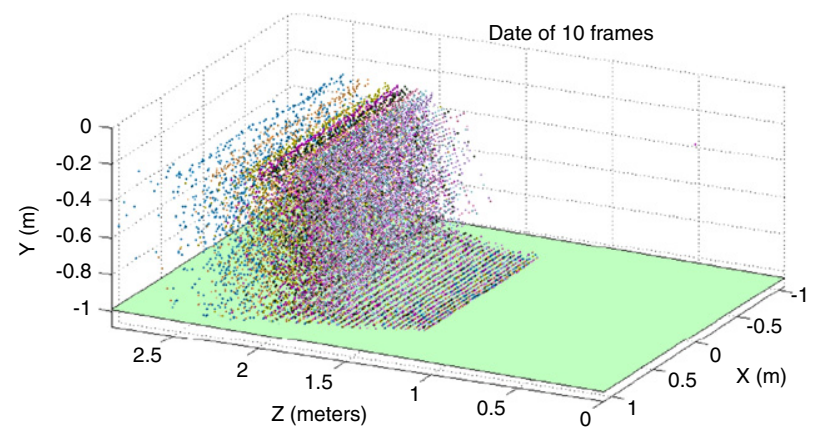

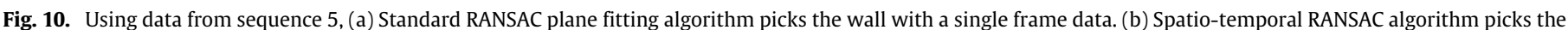
correct ground plane (10 frames). Each frame is shown in different colors.

a

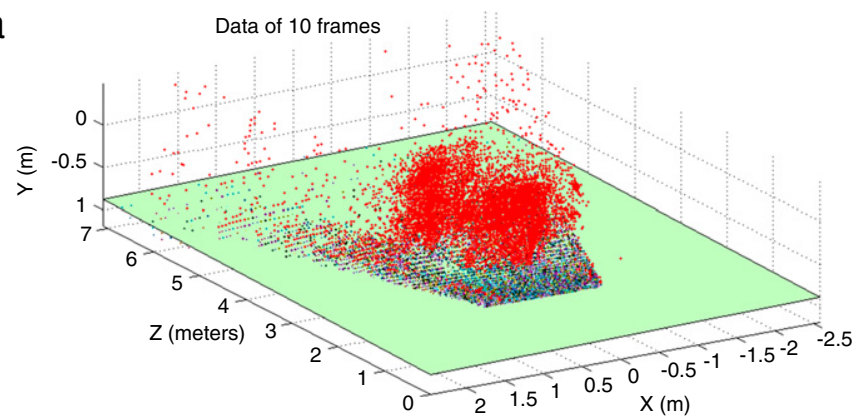

b

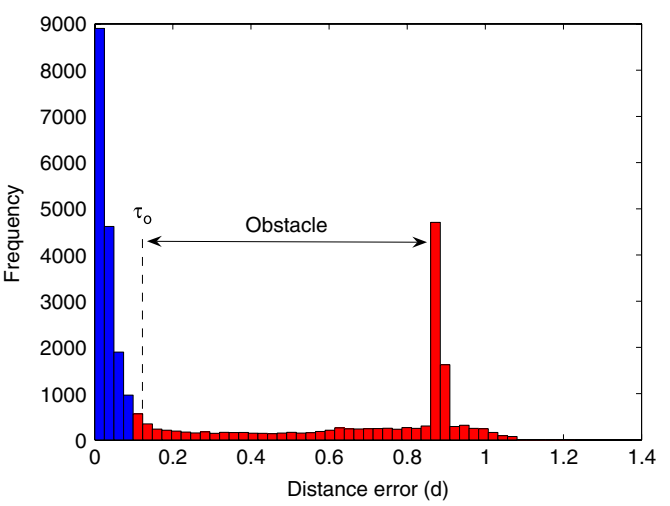

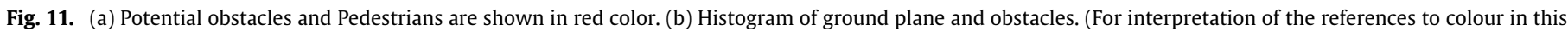
figure legend, the reader is referred to the web version of this article.)

Table 3

Number of inliers in each planar segmentation for a sequence of a specific 4D spatiotemporal data.

\begin{tabular}{lllll}
\hline \multirow{2}{*}{ Sequence no } & & \multicolumn{3}{l}{ Number of inliers } \\
\cline { 3 - 5 } \cline { 3 - 4 } 1 & Sequence & Road & Wall 1 & Wall 2 \\
\hline 5 & Double wall & 13870 & 2565 & 8964 \\
\hline
\end{tabular}

frame 4D data set consists of $3072 \times 5=15360$ data points. The run time computation cost for this example is $0.16 \mathrm{~s}$ that scales to $0.38 \mathrm{~s}$ for a ten frame data with 15156 inliers as shown in Table 4. The performance is a relative measure as it depends upon random sampling and occlusion of the planar surface that determines the inliers and outlier ratio. The efficiency of the algorithm can be gauged by the plots provided in Fig. 14 . The performance could be further improved by using a more optimized 4D RANSAC routine on $\mathrm{C}++$ platform or with FPGA implementation.

\section{Conclusion}

Many applications in the field of robotics and computer vision use some kind of segmentation and planar surface detection as a preliminary step. In this paper we have presented a robust spatiotemporal RANSAC framework for planar surface detection and segmentation with number of vision based potential applications for automotive industry and autonomous navigation. Experimental results validate the structure and motion model of a 3D spatiotemporal planar feature in 4D. Since the algorithm does not involve

Table 4

Number of inliers picked by the algorithm for each sequence for a specific 4D spatio-temporal data.

\begin{tabular}{|c|c|c|c|c|c|c|}
\hline \multirow[t]{2}{*}{ No of frames } & \multicolumn{5}{|c|}{ Number of inliers } & \multirow[t]{2}{*}{ Total data points } \\
\hline & Seq 1 & Seq 2 & Seq 3 & Seq 4 & Seq 5 & \\
\hline 5 & 6546 & 5708 & 5696 & 7461 & - & 15360 \\
\hline 6 & 8017 & 6547 & 6848 & 8739 & 5579 & 18432 \\
\hline 7 & 9390 & 7883 & 8068 & 10188 & 7228 & 21504 \\
\hline 8 & 10728 & 9068 & 9326 & 11158 & 8870 & 24576 \\
\hline 9 & 11630 & 10169 & 10153 & 13323 & 9579 & 27648 \\
\hline 10 & 13547 & 10934 & 11330 & 15156 & 11487 & 30720 \\
\hline
\end{tabular}


a

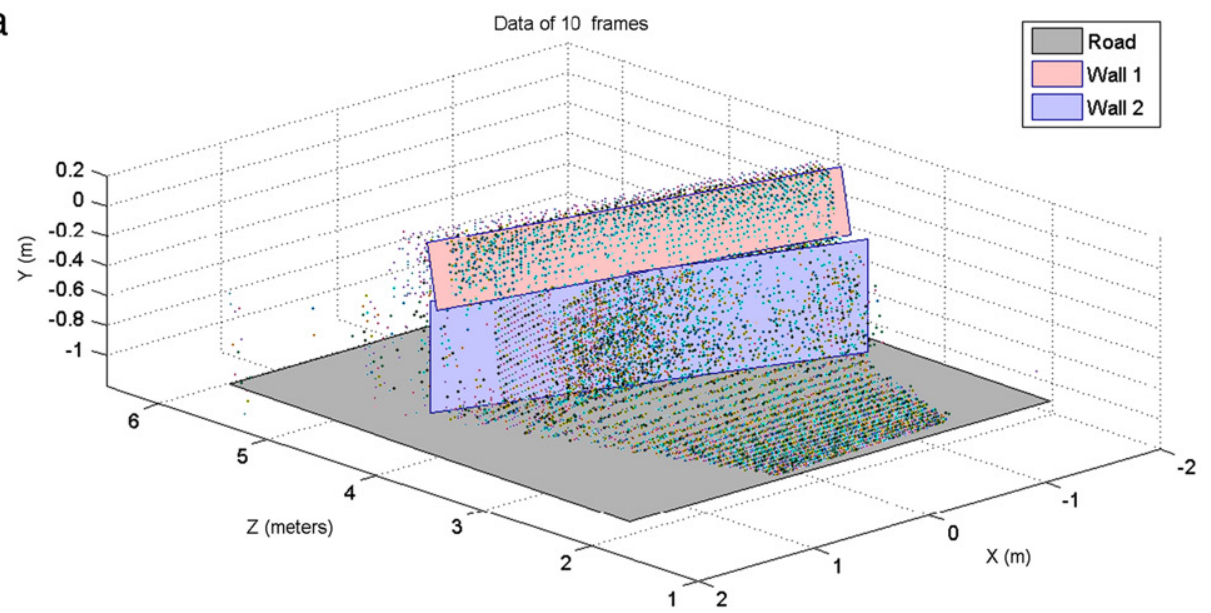

b
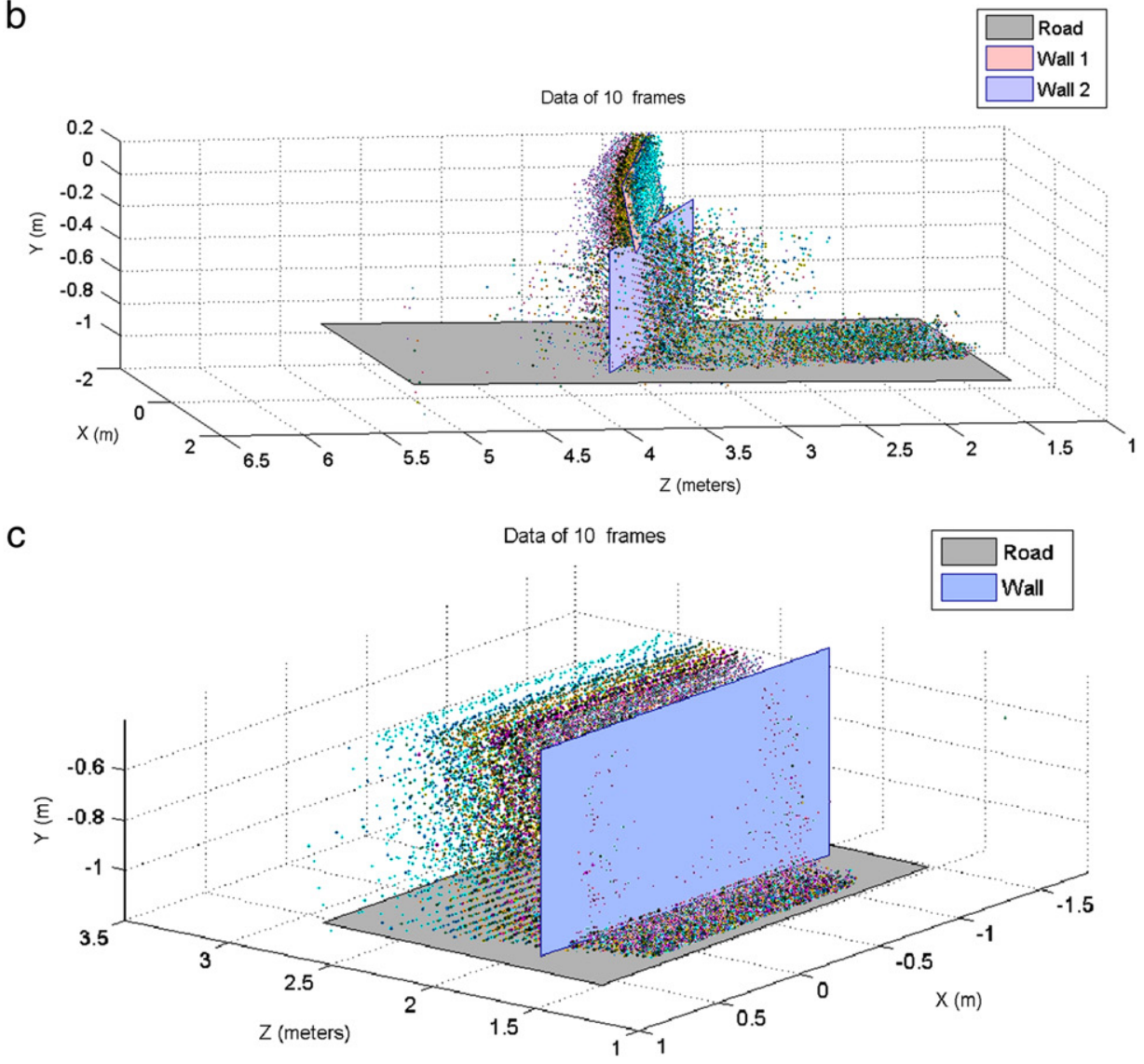

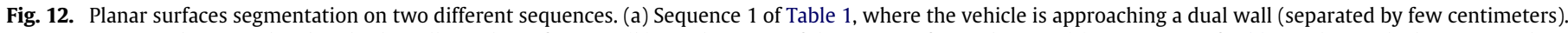

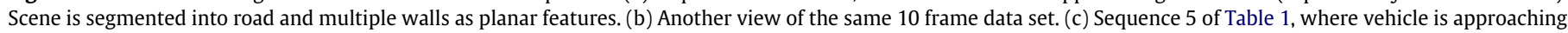
a straight wall. The algorithm has segmented scene into planar features visualized at frame $\{10\}$.

any tracking or feature selection and only requires estimation of normal vector from randomly sampled 4D data, it is highly robust, simple and practical to implement. The algorithm is suitable not only for automotive industry but also in general computer vision and robotic applications that satisfy the particular motion constraint $(\eta \times \omega=0)$. This constraint ensures that a spatial planar feature generates a planar feature in spatio-temporal domain. The spatio-temporal constraints increases reliability in planar surface estimation that is otherwise susceptible to noisy data in any algorithm developing a single frame data.
The algorithm is practical in nature and may be useful in a number of vision and navigation application domains that rely on an inexpensive segmentation and planer surfaces detection in general. Further improvement in computation cost can be achieved with dedicated hardware implementation.

\section{Acknowledgments}

This work was supported by Seeing Machines Ltd. and the Commonwealth of Australia, through the Cooperative Research Centre for Advanced Automotive Technology. 


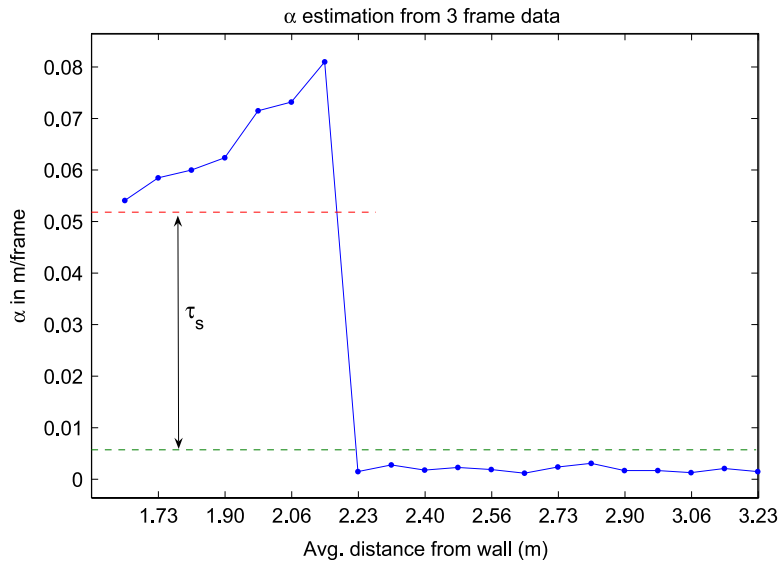

Fig. 13. Normal velocity of planar surfaces (wall and ground) with car approaching a wall and segmentation with $\tau_{s}$ on $\alpha$.

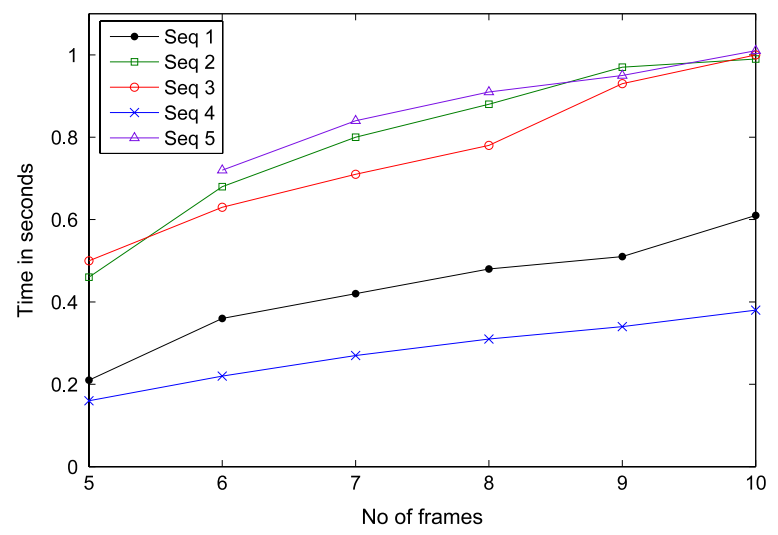

Fig. 14. Performance plots for spatio-temporal RANSAC for all the sequences.

\section{Appendix}

Given an orthonormal basis $\left\{\mathbf{e}_{1}, \ldots, \mathbf{e}_{n}\right\} \in \mathbb{R}^{n}$ the 'Levi-Civita' $(\varepsilon)$ antisymmetric tensor is defined as [74]

$$
\begin{aligned}
& \varepsilon_{i, j, \ldots, n} \\
& \quad= \begin{cases}+1 & \text { if }(i, j, \ldots, n) \text { an even permutation of }(1,2, \ldots, n) \\
-1 & \text { if }(i, j, \ldots, n) \text { an odd permutation of }(1,2, \ldots, n) \\
0 & \text { if }(i, j, \ldots, n) \text { not a permutation of }(1,2, \ldots, n) .\end{cases}
\end{aligned}
$$

The cross product of three vectors $\mathbf{a}, \mathbf{b}, \mathbf{c} \in \mathbb{R}^{4}$ is defined as [69]

$$
\operatorname{cross}_{4}(\mathbf{a}, \mathbf{b}, \mathbf{c})=(\mathbf{a} \times \mathbf{b} \times \mathbf{c})=\sum_{i, j, k, l=1}^{n=4} \varepsilon_{i j k l} a_{j} b_{k} c_{l} \mathbf{e}_{i} .
$$

The vector cross product of the three vectors in $\mathbb{R}^{4}$ has the following properties (amongst others).

1. Trilinearity: For $\alpha, \beta, \gamma \in \mathbb{R}, \alpha \mathbf{a} \times \beta \mathbf{b} \times \gamma \mathbf{c}=\alpha \beta \gamma(\mathbf{a} \times \mathbf{b} \times \mathbf{c})$.

2. Linear dependence: $\operatorname{cross}_{4}(\mathbf{a}, \mathbf{b}, \mathbf{c})=0$ iff $\langle\mathbf{a}, \mathbf{b}, \mathbf{c}\rangle$ are linearly dependent.

3. Orthogonality: Let $\mathbf{d}=\mathbf{a} \times \mathbf{b} \times \mathbf{c} \Rightarrow\langle\mathbf{d}, \mathbf{a}\rangle=\langle\mathbf{d}, \mathbf{b}\rangle=\langle\mathbf{d}, \mathbf{c}\rangle$ $=0$.

\section{References}

[1] M.W.M.G. Dissanayake, P. Newman, S. Clark, H.F. Durrant-Whyte, M. Csorba, A solution to the simultaneous localization and map building (SLAM) problem, IEEE Journal of Robotics and Automation 17 (3) (2001) 229-241. doi: $10.1109 / 70.938381$.

[2] J.-P. Laumond (Ed.), Robot Motion Planning and Control, in: Lecture Notes in Control and Information Science, vol. 229, Springer, New York, 1998.
[3] S. Lenser, M. Veloso, Visual sonar: fast obstacle avoidance using monocular vision, in: Proc. IEEE/RSJ Int. Conf. Intelligent Robots and Systems, IROS 2003, vol. 1, 2003, pp. 886-891. doi:10.1109/IROS.2003.1250741.

[4] M. Felisa, P. Zani, Incremental disparity space image computation for automotive applications, in: Proc. IEEE/RSJ Int. Conf. Intelligent Robots and Systems IROS 2009, 2009, pp. 345-350. doi:10.1109/IROS.2009.5353897.

[5] I. Rekleitis, J.-L. Bedwani, E. Dupuis, Over-the-horizon, autonomous navigation for planetary exploration, in: Proc. IEEE/RSJ Int. Conf. Intelligent Robots and Systems IROS 2007, 2007, pp. 2248-2255. doi:10.1109/IROS.2007.4399533.

[6] S.B. Kesner, J.-S. Plante, P.J. Boston, T. Fabian, S. Dubowsky, Mobility and power feasibility of a microbot team system for extraterrestrial cave exploration, in: Proc. IEEE Int. Robotics and Automation Conf., 2007, pp. 4893-4898. doi:10.1109/ROBOT.2007.364233.

[7] C. Wu, W. Tsai, Location estimation for indoor autonomous vehicle navigation by omni-directional vision using circular landmarks on ceilings, Robotics and Autonomous Systems 57 (2009) 546-555.

[8] P. Batavia, S. Singh, Obstacle detection using adaptive color segmentation and color stereo homography, in: S. Singh (Ed.), Proc. IEEE Int. Conf. Robotics and Automation, ICRA'01, vol. 1, 2001, pp. 705-710. doi:10.1109/ROBOT.2001.932633.

[9] Y. Wang, S. Fang, Y. Cao, H. Sun, Image-based exploration obstacle avoidance for mobile robot, in: Proc. Chinese Control and Decision Conf. CCDC'09, 2009, pp. 3019-3023. doi:10.1109/CCDC.2009.5192583.

[10] D. Santosh, S. Achar, C.V. Jawahar, Autonomous image-based exploration for mobile robot navigation, in: Proc. IEEE Int. Conf. Robotics and Automation ICRA 2008, 2008, pp. 2717-2722. doi:10.1109/ROBOT.2008.4543622.

[11] D. Kragic, M. Bjöorkman, H.I. Christensen, J.O. Eklundh, Vision for robotic object manipulation in domestic settings, Robotics and Autonomous Systems 52 (2005) 85-100.

[12] P.G. Zavlangas, S.G. Tzafestas, Industrial robot navigation and obstacle avoidance employing fuzzy logic, Journal of Intelligent and Robotic Systems 27 (2000) 85-97.

[13] A. Cherian, V. Morellas, N. Papanikolopoulos, Accurate 3D ground plane estimation from a single image, in: Proc. IEEE Int. Conf. Robotics and Automation ICRA'09, 2009, pp. 2243-2249. doi:10.1109/ROBOT.2009.5152260.

[14] D. Wilkie, J. van den Berg, D. Manocha, Generalized velocity obstacles, in: Proc. IEEE/RSJ Int. Conf. Intelligent Robots and Systems IROS 2009, 2009, pp. 5573-5578. doi:10.1109/IROS.2009.5354175.

[15] J. Pettre, H. Grillon, D. Thalmann, Crowds of moving objects: navigation planning and simulation, in: Proc. IEEE Int. Robotics and Automation Conf., 2007, pp. 3062-3067. doi:10.1109/ROBOT.2007.363937.

[16] S. Kim, W. Chung, C. bae Moon, J.-B. Song, Safe navigation of a mobile robot using the visibility information, in: Proc. IEEE Int Robotics and Automation Conf, 2007, pp. 1304-1309.

[17] J.H. Lee, K. Abe, T. Tsubouchi, R. Ichinose, Y. Hosoda, K. Ohba, Collision-free navigation based on people tracking algorithm with biped walking model, in: Proc. IEEE/RSJ Int. Conf. Intelligent Robots and Systems IROS 2008, 2008, pp. 2983-2989. doi:10.1109/IROS.2008.4650893.

[18] R.E. Yasovardhan, K. Hemanth, K.M. Krishna, Estimating ground and other planes from a single tilted laser range finder for on-road driving, in: Proc. Int. Conf. Advanced Robotics ICAR 2009, 2009, pp. 1-6.

[19] M. Isard, A. Blake, Condensation-conditional density propagation for visual tracking, International Journal of Computer Vision 29 (1) (1998) 5-28.

[20] P. Fornland, Direct obstacle detection and motion from spatio-temporal derivatives, in: Proc. 6th Intl. Conf. Comp. Anal. of Images and Patterns, 1995, pp. 874-879.

[21] D. Koller, K. Daniilidis, H. Nagel, Model-based object tracking in monocular image sequences of road traffic scenes, International Journal of Computer Vision 10 (3) (1993) 257-281.

[22] A. Murarka, B. Kuipers, A stereo vision based mapping algorithm for detecting inclines, drop-offs, and obstacles for safe local navigation, in: Proc. IEEE/RSJ Int. Conf. Intelligent Robots and Systems IROS 2009, 2009, pp. 1646-1653. doi:10.1109/IROS.2009.5354253.

[23] C. Shi, Y. Wang, J. Yang, A local obstacle avoidance method for mobile robots in partially known environment, Robotics and Autonomous Systems 58 (2010) 425-434.

[24] A. Rankin, A. Huertas, L. Matthies, Night-time negative obstacle detection for off-road autonomous navigation, in: SPIE, 2007.

[25] J. Larsson, M. Broxvall, A. Saffiotti, Laser based intersection detection for reactive navigation in an underground mine, in: Proc. IEEE/RSJ Int. Conf. Intelligent Robots and Systems IROS 2008, 2008, pp. 2222-2227. doi:10.1109/IROS.2008.4650911.

[26] T. Kahlmann, F. Remondino, S. Guillaume, Range imaging technology: new developments and applications for people identification and tracking, in: Proc. SPIE-IS\&T Electronic Imaging, vol. 6491, San Jose, CA, USA, 2007.

[27] K. Sobottka, H. Bunke, Vision-based driver assistance using range imagery, in: Proc. IEEE Int. Conf. Intelligent Vehicles, Stuttgart, Germany, 1998, pp. $162-167$

[28] Y.-J. Choi, J.-P. Hong, K.-W. Park, Obstacle avoidance using active window and flexible vector field with a laser range finder, in: Proc. Int. Conf. Control, Automation and Systems ICCAS'07, 2007, pp. 2123-2128. doi:10.1109/ICCAS.2007.4406681.

[29] S. May, D. Droeschel, D. Holz, S. Fuchs, E. Malis, A. Nüchter, J. Hertzberg, Threedimensional mapping with time-of-flight cameras, Journal of Field Robotics 26 (11-12) (2009) 934-965.

[30] K. Kanazawa, K. Kanatani, Reliability of fitting a plane to range data, IEICE Transactions on Information and Systems E78-D (12) (1995) 1630-1635. 
[31] D. Watanabe, H. Saito, Planar structure based registration of multiple range images, in: Proc. 18th International Conference on Pattern Recognition ICPR 2006, vol. 3, 2006, pp. 689-692. doi:10.1109/ICPR.2006.935.

[32] E. Kim, S. Medioni, G. Lee, Planar patch based 3D environment modeling with stereo camera, in: Proc. 16th IEEE Int. Symp. Robot and Human Interactive Communication, Jeju Island, Korea, 2007.

[33] S. Lee, D. Jang, E. Kim, S. Hong, J. Han, A real-time 3D workspace modeling with stereo camera, in: Proc. IEEE/RSJ Int. Conf. Intelligent Robots and Systems, IROS'05, 2005, pp. 2140-2147. doi:10.1109/IROS.2005.1545105.

[34] F. Schmitt, X. Chen, Fast segmentation of range images into planar regions, in: Proc. CVPR'91. IEEE Computer Society Conference on Computer Vision and Pattern Recognition, 1991, pp. 710-711. doi:10.1109/CVPR.1991.139793.

[35] X. Jiang, H. Bunke, Fast segmentation of range images into planar regions by scan line grouping, Machine Vision and Applications 7 (1994) 115-122.

[36] E.B. Meier, F. Ade, Object detection and tracking in range image sequences by separation of image features, in: Proc. IEEE Int. Conf. Intelligent Vehicles, 1998.

[37] C. Wang, H. Tanahashi, H. Hirayu, Y. Niwa, K. Yamamoto, Comparison of local plane fitting methods for range data, in: Proc. IEEE Conf. Computer Vision and Pattern Recognition, CVPR'01, 2001.

[38] D. Hähnel, W. Burgard, S. Thrun, Learning compact 3D models of indoor and outdoor environments with a mobile robot, Robotics and Autonomous Systems 44 (1) (2003) 15-27.

[39] D. Wolf, A. Howard, G.S. Sukhatme, Towards geometric 3D mapping of outdoor environments using mobile robots, in: Proc. IEEE/RSJ International Conference on Intelligent Robots and Systems, IROS 2005, 2005, pp. 1507-1512. doi:10.1109/IROS.2005.1545152.

[40] S. Thrun, C. Martin, Y. Liu, D. Hahnel, R. Emery-Montemerlo, D. Chakrabarti, W. Burgard, A real-time expectation-maximization algorithm for acquiring multiplanar maps of indoor environments with mobile robots, 20 (3) 2004 433-443. doi:10.1109/TRA.2004.825520.

[41] Y. Liu, R. Emery, D. Chakrabarti, W. Burgard, S. Thrun, Using EM to learn 3D models of indoor environments with mobile robots, in: Proc. 18th Int. Conf. Machine Learning, 2001, pp. 329-336.

[42] C. Martin, S. Thrun, Real-time acquisition of compact volumetric 3D maps with mobile robots, in: Proc. IEEE International Conference on Robotics and Automation ICRA'02, vol. 1, 2002, pp. 311-316. doi:10.1109/ROBOT.2002.1013379.

[43] R. Triebel, W. Burgard, F. Dellaert, Using hierarchical EM to extract planes from 3D range scans, in: Proc. IEEE International Conference on Robotics and Automation ICRA 2005, 2005, pp. 4437-4442.

[44] R. Lakaemper, L.J. Latecki, Using extended EM to segment planar structures in 3D, in: Proc. 18th International Conference on Pattern Recognition ICPR 2006, vol. 3, 2006, pp. 1077-1082. doi:10.1109/ICPR.2006.1154.

[45] H. Cantzler, R.B. Fisher, M. Devy, Improving architectural 3D reconstruction by plane and edge constraining, in: Proc. British Machine Vision Conf. BMVC'02, 2002, pp. 43-52.

[46] A. Nüchter, H. Surmann, J. Hertzberg, Automatic model refinement for 3D reconstruction with mobile robots, in: Proc. Fourth Int. Conf. 3-D Digital Imaging and Modeling, 3DIM'03, 2003, pp. 394-401.

[47] M. Perrollaz, R. Labayrade, C. Royere, N. Hautiere, D. Aubert, Long range obstacle detection using laser scanner and stereovision, in: $R$. Labayrade (Ed.), Proc. IEEE Intelligent Vehicles Symp. 2006, pp. 182-187. doi:10.1109/IVS.2006.1689625

[48] H. Isack, Y. Boykov, Energy-based geometric multi-model fitting, Tech. Rep. 735, University of Western Ontario, Canada, March 2010

[49] P. Labatut, J.-P. Pons, R. Keriven, Hierarchical shape-based surface reconstruction for dense multi-view stereo, in: Proc. IEEE 12th Int. Computer Vision Workshops, ICCV Workshops, Conf., 2009, pp. 1598-1605. doi:10.1109/ICCVW.2009.5457421.

[50] F. Kahl, A. Heyden, L. Quan, Minimal projective reconstruction including missing data, IEEE Transactions on Pattern Analysis and Machine Intelligence 23 (4) (2001) 418-424. doi:10.1109/34.917578.

[51] T. Moller, H. Kraft, J. Frey, M. Albrecht, R. Lange, Robust 3D measurement with PMD sensors, in: Proc. 1st Range Imaging Research Day, ETH Zurich, Zurich, Switzerland, 2005

[52] J. Poppinga, N. Vaskevicius, A. Birk, K. Pathak, Fast plane detection and polygonalization in noisy 3D range images, in: Proc. IEEE/RS Int. Conf. Intelligent Robots and Systems IROS 2008, 2008, pp. 3378-3383. doi:10.1109/IROS.2008.4650729.

[53] G.-P.M. Hegde, C. Ye, Extraction of planar features from swissranger SR-3000 range images by a clustering method using normalized cuts, in: Proc. IEEE/RSJ Int. Conf. Intelligent Robots and Systems IROS 2009, 2009, pp. 4034-4039. doi:10.1109/IROS.2009.5353952.

[54] R.C. Bolles, M.A. Fischler, A RANSAC-based approach to model fitting and its application to finding cylinders in range data, in: Proc. Seventh Int. Joint Conf. Artificial Intelligence, 1981, pp. 637-643.

[55] F. Mufti, R. Mahony, J. Heinzmann, Spatio-temporal RANSAC for robust estimation of ground plane in video range images for automotive applications, in: Proc. IEEE Intell. Transp. Syst. Conf. ITSC'08, 2008, pp. 1142-1148.

[56] A. Bartoli, Piecewise planar segmentation for automatic scene modeling, in: Proc. IEEE Int. Conf. Computer Vision and Pattern Recognition, CVPR'01, 2001.

[57] Z. Hongsheng, S. Negahdaripour, Improved temporal correspondences in stereo-vision by RANSAC, in: Proc. 17th Int. Conf. Pattern Recognition, ICPR'04, vol. 4, 2004, pp. 52-55.

[58] D.-S. Tue-Cuong, G. Dong, Y.C. Hwang, O.S. Heng, Robust extraction of shady roads for vision-based UGV navigation, in: Proc. IEEE/RSJ Int. Conf. Intelligent Robots and Systems IROS 2008, 2008, pp. 3140-3145. doi:10.1109/IROS.2008.4650955.
[59] A. Yang, S. Rao, Y. Ma, Robust statistical estimation and segmentation of multiple subspaces, in: Proc. Conference on Computer Vision and Pattern Recognition Workshop CVPRW'06, 2006, pp. 99-99. doi:10.1109/CVPRW.2006.178.

[60] J. Lam, K. Kusevic, R. Mrstik, P. Harrap, M. Greenspan, Urban scene extraction from mobile ground based lidar data, in: Proc. 3DPVT 2011, 2010.

[61] T. Spirig, M. Marley, P. Seitz, The multitap lock-in CCD with offset subtraction, IEEE Transactions on Electron Devices 44 (10) (1997) 1643-1647.

[62] IEEE(CVPR), IEEE Conf. Computer Vision and Pattern Recognition, CVPR, Workshop on TOF Camera based Computer Vision, TOF-CV.

[63] M.A. Fischler, R.C. Bolles, Random sample consensus: a paradigm for mode fitting with applications to image analysis and automated cartography, Communications of the ACM 24 (6) (1981) 381-395.

[64] Z. Xu, R. Schwarte, H. Heinol, B. Buxbaum, T. Ringbeck, Smart pixel-photonic mixer device (PMD), new system concept of a 3D-imaging camera-on-a-chip, in: 5th Int. Conf. Mechatronics and Machine Vision in Practice, Nanjing, 1998, pp. 259-264.

[65] T. Spirig, P. Seitz, O. Vietze, F. Heitger, The lock-in CCD-two dimensional synchronous detection of light, IEEE Journal of Quantum Electronics 31 (9) (1995) 1705-1708.

[66] J.J. Craig, Introduction to Robotics: Mechanics and Control, Prentice Hall, 2003.

[67] G.A. Gracia, F. Jimenez, J. Paez, A. Narvaez, Theoretical and experimental analysis to determine the influence of the ageing process of the shock-absorber on safety, International Journal of Vehicle Design 40 (1-2-3) (2006) 15-35.

[68] G.D. Sullivan, Real-Time Computer Vision, Cambridge University Press, Cambridge, 1994 (Chapter) Model-based vision for traffic scenes using the ground plane constraint, pp. 93-115.

[69] R. Shaw, Vector cross products in $n$ dimensions, International Journal of Mathematical Education in Science and Technology 18 (6) (1987) 803-816.

[70] F. Mufti, R. Mahony, Statistical analysis of measurement processes for time-offlight cameras, in: Proc. SPIE Videometrics, Range Imaging, and Applications X, vol. 7447-21, 2009.

[71] R. Hartley, A. Zisserman, Multiple View Geometry in Computer Vision, Cambridge University Press, 2003 (Chapter) Estimatio-2D Projective Transformation, pp. 118.

[72] O. Wulf, C. Brenneke, B. Wagner, Colored 2D maps for robot navigation with 3D sensor data, in: Proc. IEEE/RSJ Int. Conf. Intelligent Robots and Systems, IROS 2004, vol. 3, 2004, pp. 2991-2996. doi:10.1109/IROS.2004.1389864.

[73] T. Ringbeck, A 3D time-of-flight camera for object detection, in: Proc. Optical 3-D measurement Techniques, 2007.

[74] C.W. Misner, K.S. Thorne, J.A. Wheeler, Gravitation, W.H. Freeman, San Francisco, CA, 1973, p. 87.

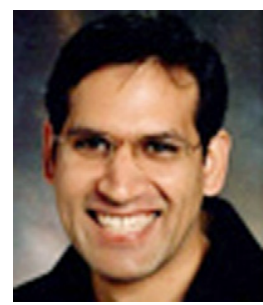

Faisal Mufti is Assistant Professor at Center for Advanced Studies in Engineering (CASE), Islamabad, Pakistan. He received his Ph.D. in Computer Vision from the Australian National University (ANU) in 2010. His main area of research includes vision based automotive applications using time-of-flight (TOF) cameras. His publishing area includes super resolution (won best student paper award at DICTA 2007), segmentation, statistical analysis and radiometric and reflectance modeling using 3D TOF camera. He has also served as reviewer on the panel for IEEE Transactions on Intelligent Transportation Systems and Journal of Mathematical Imaging and Vision.

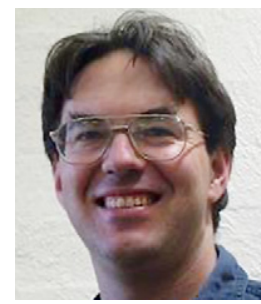

Robert Mahony is currently a reader (Associate Professor) in the Department of Engineering at the Australian National University. He received a Ph.D. in 1995 (systems engineering) and a B.Sc. in 1989 (applied mathematics and geology) both from the Australian National University. He worked as a marine seismic geophysicist and an industrial research scientist before completing a postdoctoral fellowship in France and a Logan Fellowship at Monash University in Australia. He has held his post at ANU since 2001 . His research interests are in non-linear control theory with applications in robotics, geometric optimization techniques and systems theory.

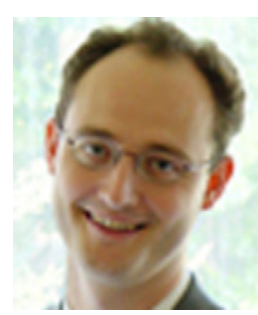

Jochen Heinzmann received his Ph.D in human robot interaction from The Australian National University in 2001. He then co-founded Seeing Machines Limited in Canberra, which specializes in computer vision facial image processing applications. Jochen still works at Seeing Machines and his research interests include aspects of facial image processing, detection and management of fatigue in operators and drivers and sleep physiology. 